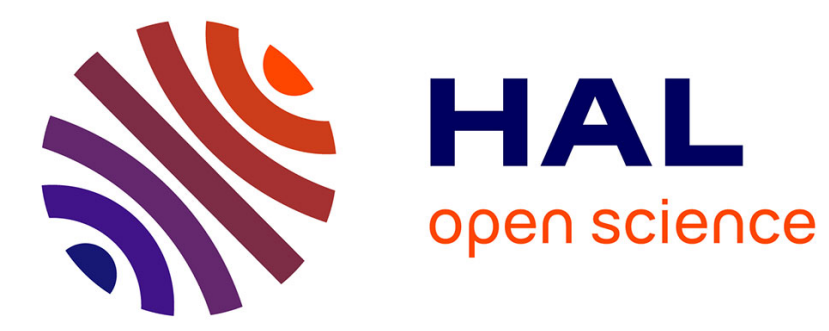

\title{
Turbulent transport and entrainment in jets and plumes: A DNS study
}

Maarten R van Reeuwijk, Pietro R Salizzoni, Gary R Hunt, John Craske

\section{To cite this version:}

Maarten R van Reeuwijk, Pietro R Salizzoni, Gary R Hunt, John Craske. Turbulent transport and entrainment in jets and plumes: A DNS study. Physical Review Fluids, 2016, 1, pp.074301. 10.1103/PhysRevFluids.1.074301 . hal-01516094

\section{HAL Id: hal-01516094 \\ https://hal.science/hal-01516094}

Submitted on 4 Jul 2017

HAL is a multi-disciplinary open access archive for the deposit and dissemination of scientific research documents, whether they are published or not. The documents may come from teaching and research institutions in France or abroad, or from public or private research centers.
L'archive ouverte pluridisciplinaire HAL, est destinée au dépôt et à la diffusion de documents scientifiques de niveau recherche, publiés ou non, émanant des établissements d'enseignement et de recherche français ou étrangers, des laboratoires publics ou privés. 


\title{
Turbulent transport and entrainment in jets and plumes: A DNS study
}

\author{
Maarten van Reeuwijk* \\ Department of Civil and Environmental Engineering, Imperial College London, \\ London SW7 2AZ, United Kingdom
}

Pietro Salizzoni

Laboratoire de Mécanique des Fluides et d'Acoustique, University of Lyon, CNRS UMR No. 5509, Ecole Centrale de Lyon, INSA Lyon, Université Claude Bernard, 36 Avenue Guy de Collongue, 69134 Ecully, France

Gary R. Hunt

Department of Engineering, University of Cambridge, Cambridge CB2 1PZ, United Kingdom

John Craske

Department of Civil and Environmental Engineering, Imperial College London, London SW7 2AZ, United Kingdom

(Received 31 March 2016; published 7 November 2016)

\begin{abstract}
We present a direct numerical simulation (DNS) data set for a statistically axisymmetric turbulent jet, plume, and forced plume in a domain of size $40 r_{0} \times 40 r_{0} \times 60 r_{0}$, where $r_{0}$ is the source diameter. The data set supports the validity of the Priestley-Ball entrainment model in unstratified environments (excluding the region near the source) [Priestley and Ball, Q. J. R. Meteor. Soc. 81, 144 (1955)], which is corroborated further by the Wang-Law and Ezzamel et al. experimental data sets [Wang and Law, J. Fluid Mech. 459, 397 (2002); Ezzamel et al., ibid. 765, 576 (2015)], the latter being corrected for a small but influential coflow that affected the statistics. We show that the second-order turbulence statistics in the core region of the jet and the plume are practically indistinguishable from each other, although there are significant differences near the plume edge. The DNS data indicate that the turbulent Prandtl number is about 0.7 for both jets and plumes. For plumes, this value is a result of the difference in the ratio of the radial turbulent transport of radial momentum and buoyancy. For jets, however, the value originates from a different spread of the buoyancy and velocity profiles, in spite of the fact that the ratio of radial turbulent transport terms is approximately unity. The DNS data do not show any evidence of similarity drift associated with gradual variations in the ratio of buoyancy profile to velocity profile widths.
\end{abstract}

DOI: 10.1103/PhysRevFluids.1.074301

\section{INTRODUCTION}

The mixing of buoyant fluid releases with the surrounding fluid is of primary concern for a wide number of industrial and environmental turbulent flows, spanning the ascending motions of thermals in the atmosphere, the rise and fall of volcanic eruption columns, the release of airborne pollutants, or the propagation of smoke in free or enclosed spaces [1]. Much attention has therefore been paid to the turbulence dynamics of buoyant releases in a multiplicity of flow configurations. One of the most studied flows among these, commonly referred to as a plume, is the free-shear flow arising from a localized source of buoyancy. Since the pioneering work of Zel'dovich [2], Priestley and Ball [3], and Morton et al. [4], plumes have been the subject of several theoretical [5], experimental [6-9], and

*m.vanreeuwijk@imperial.ac.uk; civilsfluids@imperial.ac.uk 
numerical [10,11] investigations and are well documented in a number of review articles [12-14]. In this context, the well-known turbulent jet can be regarded as a plume without buoyancy and provides a reference state for understanding how buoyancy modifies the behavior of these free-shear flows.

Jets and plumes are canonical examples of flows that evolve in a self-similar fashion [14]: Sufficiently far from the source, a rescaling of the radial coordinate and dependent variables by a characteristic local width $r_{m}$, velocity $w_{m}$, and buoyancy $b_{m}$ results in a collapse of the data onto a single curve. The velocity and buoyancy profiles are well represented by a Gaussian form [12] and self-similarity allows power laws, relating the scales $r_{m}, w_{m}$, and $b_{m}$ to the streamwise (vertical direction opposing the gravitational vector) $z$ coordinate [4], to be deduced. Due to the presence of buoyancy, the $z$ dependence of plumes is markedly different from that of jets, yet in other respects, as discussed in this paper, these flows are broadly alike.

There are several ways to determine the characteristic scales $r_{m}, w_{m}$, and $b_{m}$. A popular experimental method is to capitalize on the Gaussian shape of the velocity and buoyancy profiles and associate $r_{m}$ with the standard deviation of the Gaussian and $w_{m}$ and $b_{m}$ with the maximum velocity and buoyancy, respectively. A method that does not rely directly on the assumption of a Gaussian shape is to determine local scales based on integral quantities of the flow:

$$
r_{m} \equiv \frac{Q}{M^{1 / 2}}, \quad w_{m} \equiv \frac{M}{Q}, \quad b_{m} \equiv \frac{B}{r_{m}^{2}},
$$

where the integral volume flux $Q$, specific momentum flux $M$, and buoyancy $B$ are defined as

$$
Q \equiv 2 \int_{0}^{\infty} \bar{w} r d r, \quad M \equiv 2 \int_{0}^{\infty} \bar{w}^{2} r d r, \quad B \equiv 2 \int_{0}^{\infty} \bar{b} r d r .
$$

Here $\bar{w}$ is the average (ensemble or time) streamwise velocity, $b=g\left(\rho_{e}-\rho\right) / \rho_{e}$ is the fluid buoyancy and $\bar{b}$ its average value, $g$ is the modulus of the gravitational acceleration, and $\rho_{e}$ is the density of the environment. Here $Q, M$, and $B$ are scaled, rather than actual, integral fluxes due to a factor $\pi$ that is not present in their definitions; this is common practice as it simplifies the resulting analytical expressions [15].

It should be noted that the definition of $b_{m}$, in Eq. (1) is nonstandard as it is usually expressed in terms of the buoyancy flux

$$
F \equiv 2 \int_{0}^{\infty} \bar{w} \bar{b} r d r
$$

as $b_{m}=F / Q=F / w_{m} r_{m}^{2}$. While this is a perfectly reasonable definition, it implicitly assumes averaging over a radius associated with the buoyancy profile that, in general, will not be exactly equal to $r_{m}$. With a single length scale $r_{m}$ as defined in Eq. (1), it follows that $F=\theta_{m} w_{m} r_{m}^{2} b_{m}$, where $\theta_{m}$ is a dimensionless profile coefficient (see also Sec. III C); thus the definition of $b_{m}$ in terms of $F$, in the current framework, is $b_{m}=F / \theta_{m} Q$. The profile coefficient $\theta_{m}$, which is intimately related to the ratio of the widths of the buoyancy and velocity profiles (see Sec. IIIC), plays an important role in longitudinal mixing in jets [16], and is purportedly responsible for the large scatter in measurements of plume entrainment [17].

The dilution of jets and plumes can be quantified by integrating the continuity equation over the radial direction, which results in

$$
\frac{1}{r_{m}} \frac{d Q}{d \zeta}=-2[r u]_{\infty}
$$

Here $\zeta \equiv \int_{0}^{z} r_{m}^{-1} d z^{\prime}$ is a dimensionless vertical coordinate and $[r u]_{\infty}$ is a net volume flux into the jet or plume per unit height. The entrainment assumption [4,18-20] links the radial volume flux to internal jet or plume properties via

$$
-[r u]_{\infty}=\alpha r_{m} w_{m}
$$


where $\alpha$ is the entrainment coefficient. Substitution of (5) into (4) and rearranging results in

$$
\alpha=\frac{1}{2 Q} \frac{d Q}{d \zeta}
$$

Thus, the entrainment coefficient can be interpreted as (half) the relative increase in volume flux over a typical jet or plume radius $r_{m}$. This relation also clearly establishes that $\alpha$ is a measure of dilution: the higher its value, the more fluid will be mixed into the jet or plume per (vertical) unit $r_{m}$.

Typical ranges of values for $\alpha$ in jets and plumes are, respectively [21], $0.065<\alpha_{j}<0.084$ and $0.10<\alpha_{p}<0.16$, which, in spite of the scatter, strongly suggests that $\alpha_{p}>\alpha_{j}$. Using the observation that the spreading rates $d r_{m} / d z$ of jets and plumes are approximately equal [12,22] and the well-known far-field solutions $r_{m}=2 \alpha_{j} z$ and $r_{m}=\frac{6}{5} \alpha_{p} z$ for jets [23] and plumes [4], respectively, it follows directly that

$$
\alpha_{p} \approx \frac{5}{3} \alpha_{j}
$$

By applying the relation above to the observed range of values of $\alpha_{j}$, we obtain $0.108<5 \alpha_{j} / 3<$ 0.133 , which is in reasonably good agreement with the available data for $\alpha_{p}$.

The fact that the spreading rates of jets and plumes are practically identical is intimately linked with the turbulence production in the interior. Indeed, by considering balance equations for the kinetic energy of the mean flow in jets and plumes [17,24-26], the spreading rate can be directly linked to the turbulence production inside the plume. For a self-similar Gaussian plume, ignoring turbulence and pressure effects and assuming $\theta_{m}=1$, it follows that [26]

$$
\frac{d r_{m}}{d z}=-\frac{3}{4} \delta_{m}
$$

where

$$
\delta_{m}=\frac{4}{w_{m}^{3} r_{m}} \int_{0}^{\infty} \overline{u^{\prime} w^{\prime}} \frac{d \bar{w}}{d r} r d r
$$

is a dimensionless profile coefficient associated with the integral of turbulence production due to shear. This quantity is generally negative as it signifies the energy transfer from the mean to the turbulence. Hence, under the realistic assumptions leading to (8), it follows that $\delta_{m}$ is solely responsible for the plume spread and identical spreading rates imply identical values for $\delta_{m}$. Direct estimations, either using flow measurements or with high-fidelity simulations, confirm that the value of $\delta_{m}$ for jets and plumes is indeed nearly identical [26].

Using the equation for mean kinetic energy, it is possible to derive entrainment relations that fundamentally link $\alpha$ to the production of turbulence kinetic energy, the Richardson number, and shape effects. For a self-similar Gaussian plume with $\theta_{m}=1$, ignoring turbulence and pressure effects [24], the entrainment relation is [26]

$$
\alpha=-\frac{3}{8} \delta_{m}+\frac{1}{4} \mathrm{Ri},
$$

where the Richardson number Ri, defined as

$$
\mathrm{Ri}=\frac{b_{m} r_{m}}{w_{m}^{2}}=\frac{B Q}{M^{3 / 2}},
$$

characterizes the significance of buoyancy compared with inertia. An important implication of the fact that $\delta_{m}$ does not differ between jets and plumes is that (10) shows that the difference in $\alpha$ is caused purely by the influence of mean buoyancy via Ri. By using the observation that $\delta_{m}$ is a constant, (10) can be rewritten as [26]

$$
\alpha=\alpha_{j}+\left(\alpha_{p}-\alpha_{j}\right) \Gamma,
$$

which is commonly referred to as the Priestley-Ball entrainment model [3,24]. Here $\Gamma=\mathrm{Ri} / \mathrm{Ri}_{p}$ is the flux balance parameter, where $\mathrm{Ri}_{p}=8 \alpha_{p} \beta_{g} / 5$ is the Richardson number for a pure plume [26] 
and $\beta_{g}$ is a profile coefficient associated with the total momentum flux (see Sec. IIIC for its definition). The condition $\Gamma=1$ represents a stable equilibrium (with respect to perturbations in $\Gamma$ ), a condition referred to as that of a pure plume. The other equilibrium condition is given by $\Gamma=0$, i.e., that of a pure jet, which is a condition that is unstable to the addition of an arbitrarily small amount of buoyancy [15]. For forced plumes, which have an excess of momentum (relative to pure plume conditions) at the source [5], $0<\Gamma<1$, whereas $\Gamma>1$ for lazy plumes, which have a deficit of momentum [15]. Previous experimental studies observed that (12) accurately describes the behavior of jets, plumes, and forced plumes [9,25].

If the magnitude of the dimensionless turbulence production $\delta_{m}$ is approximately equal in jets and plumes, one is led to ask what this implies about the radial transport of scalar quantities in the flow. The turbulent Prandtl number

$$
\operatorname{Pr}_{T}=\frac{v_{T}}{D_{T}}
$$

where $v_{T}$ and $D_{T}$ are the eddy viscosity and eddy diffusivity, respectively, quantifies the effectiveness with which the flow mixes momentum compared with buoyancy or mass and is a useful quantity in this regard. The consensus is that $\operatorname{Pr}_{T}=0.7$ in axisymmetric jets and plumes [27], which suggests that turbulence transports buoyancy or mass more efficiently than momentum [28] in both cases. However, the underlying physics and their implications for entrainment and for the relative widths of the scalar profile compared with the velocity profile are not understood. For jets there is good agreement between investigators that suggests the scalar field is wider than the velocity field $[6,9,23,27]$. For plumes, however, as discussed in Ref. [29] and elsewhere, there is significant uncertainty: some studies reveal that the velocity field is wider than the buoyancy field $[8,27,30]$, others reveal that it is narrower $[6,23,25,31]$; several results imply that the velocity and scalar profiles have roughly the same width $[9,32]$ and some imply that the relative width varies with height [17]. The present paper seeks to untangle the confusion regarding the relationship between $\operatorname{Pr}_{T}$ and the widths of the scalar and velocity profiles by supplementing the available experimental data with precise information from direct numerical simulation (DNS).

Herein, we follow the approach of Ezzamel et al. [25] by performing a side-by-side comparison of turbulent jets, plumes, and the intermediate case of a forced plume, but using DNS rather than laboratory experiments. With DNS it is relatively straightforward to prescribe boundary conditions consistent with the analytical solutions and furthermore, DNS provides access to all variables, including pressure, at Kolmogorov-scale resolutions. In Sec. II the simulation details are presented. Integral flow statistics, such as the evolution of $\Gamma(z)$, are presented in Sec. III A and the deduced entrainment coefficient $\alpha$ is shown to follow closely the Priestley-Ball entrainment model (12). Self-similarity of the first- and second-order statistics is discussed in Sec. III B, which includes an analysis of the invariants of the anisotropy tensor. Profile coefficients, which represent the relative contribution of various physical processes relative to the characteristic scales, are presented in Sec. III C and these are used to decompose the entrainment coefficient into its individual components in Sec. III D. Section III E discusses the radial turbulent transport of streamwise momentum and buoyancy, as quantified by the eddy viscosity $v_{T}$ and diffusivity $D_{T}$. The turbulent Prandtl number will be decomposed and it is shown that even though jets and plumes share a very similar value for $\mathrm{Pr}_{T}$, the underlying reason in each case is different. Concluding remarks are made in Sec. IV.

\section{SIMULATION DETAILS}

We simulate axisymmetric jets and plumes driven by an isolated source of steady specific momentum flux $M_{0}$, volume flux $Q_{0}$, and buoyancy flux $F_{0}$. The source is approximately circular and located at the center of the base of a cuboidal domain of size $40^{2} \times 60$ source radii $r_{0}$. The fluid motion is governed by the incompressible Navier-Stokes equations under the Boussinesq approximation, which we solve numerically using $1280^{2} \times 1920$ computational cells over a uniform Cartesian grid. The code for the DNS employs a spatial discretization of fourth-order accuracy that 
TABLE I. Simulation details. The entrainment coefficient $\alpha$ and virtual origin $z_{v}$ are determined directly from $r_{m}$ (see Fig. 1). The constants $a_{w}$ and $a_{b}$ are prefactors of the mixing lengths of velocity and buoyancy, respectively [Eq. (25)], and $\left\langle\operatorname{Pr}_{T}\right\rangle$ is the typical turbulent Prandtl number [Eq. (13)].

\begin{tabular}{lcccccccccc}
\hline \hline Simulation & $N_{x} N_{y} N_{z}$ & $L_{x} L_{y} L_{z} / r_{0}^{3}$ & $\mathrm{Re}_{0}$ & $\Gamma_{0}$ & $t_{\text {run }} / \tau_{0}$ & $\alpha$ & $z_{v} / r_{0}$ & $a_{w}$ & $a_{b}$ & $\left\langle\operatorname{Pr}_{T}\right\rangle$ \\
\hline $\mathrm{J}$ & $1280^{2} \times 1920$ & $40^{2} \times 60$ & 5000 & 0 & 400 & 0.067 & -3.66 & 0.12 & 0.14 & 0.72 \\
$\mathrm{~F}$ & $1280^{2} \times 1920$ & $40^{2} \times 60$ & 5000 & $\approx 0.03$ & 480 & varies & varies & & & \\
$\mathrm{P}$ & $1280^{2} \times 1920$ & $40^{2} \times 60$ & 1667 & $\infty$ & 480 & 0.105 & -3.90 & 0.13 & 0.15 & 0.68 \\
\hline \hline
\end{tabular}

conserves volume, momentum, and energy, and integration in time is performed using a third-order Adams-Bashforth scheme (further details can be found in Ref. [33]). On the vertical and top faces of the domain we impose open boundary conditions. These allow fluid to enter and leave the domain in a manner that is consistent with flow in an unconfined domain [34]. We initiate the turbulence by applying uncorrelated perturbations of $1 \%$ to the velocities in the first cell above the source.

To simulate the jet $\mathrm{J}$ we impose a constant uniform vertical velocity $w_{0}$ at the source. Consequently, a constant scalar flux can be maintained by imposing a Dirichlet boundary condition $b=b_{0}$ on a given scalar quantity $b$ at the source. For the jet simulation $\mathrm{J}$, this scalar quantity is passive, i.e., its presence does not imply a source term in the momentum equation. In the forced plume simulation $\mathrm{F}$, for which $b$ corresponds to buoyancy, the Dirichlet boundary condition on $b$ at the source results in a positive buoyancy flux $F_{0}$. The source conditions used in the simulation of plume $\mathrm{P}$ correspond to $w_{0}=0$ and a specified positive integral buoyancy flux $F_{0}$; in practice, the buoyancy flux $F_{0}$ is a diffusive flux resulting from a Neumann condition on the buoyancy at the source. Therefore, the plume simulation $\mathrm{P}$ is infinitely lazy at the source $\left(\Gamma_{0} \equiv 5 F_{0} Q_{0}^{2} / 8 \alpha_{p} M_{0}^{5 / 2}=\infty\right)$ although, over a relatively short distance, plume $\mathrm{P}$ becomes pure. Based on the analysis of Hunt and Kaye [15], in which a constant entrainment coefficient model is assumed, the rate of decrease of the local Richardson number immediately above a highly lazy plume source scales as

$$
\left.\frac{d \Gamma}{d \zeta}\right|_{\zeta=0} \propto-\Gamma_{0}^{9 / 5}
$$

Thus, the vertical distance required to approach pure-plume behavior reduces to zero as the laziness of the source increases, i.e., as $\Gamma_{0} \rightarrow \infty$. As a consequence, our plume arising from the heated disk boundary condition, which represents the limit of an infinitely lazy plume source, is expected to establish pure-plume behavior immediately above the source and as such to closely mimic a true pure-plume source. For jet $\mathrm{J}$ and forced plume $\mathrm{F}$ we define the source Reynolds number $\operatorname{Re}_{0} \equiv 2 M_{0}^{1 / 2} / \nu$ and for plume $\mathrm{P}, \operatorname{Re}_{0} \equiv 2 F_{0}^{1 / 3} r_{0}^{2 / 3} / \nu$. The calculated values of $\mathrm{Re}_{0}$, in addition to further details of the simulations, can be found in Table I.

Statistics were acquired from each simulation over a duration that is large in comparison with the typical turnover time. For jet $\mathrm{J}$ and forced plume $\mathrm{F}$, the turnover time based on the source conditions is $\tau_{0} \equiv r_{0}^{2} / M_{0}^{1 / 2}$. For plume $\mathrm{P}, \tau_{0} \equiv r_{0}^{4 / 3} / F_{0}^{1 / 3}$. Prior to obtaining statistics we ensure that transient effects arising from initial conditions are imperceptible in the leading-order statistics. Statistics were gathered over the time period shown in Table I.

Azimuthally averaged data were obtained by partitioning the domain into concentric cylindrical cells and averaging over all cells lying within a given shell. To compute integrals over lateral slices of the jet (for the definition of these integrals see Sec. III C), we define the upper limit of integration $r_{d}$ according to $\bar{w}\left(r_{d}, z, t\right)=0.02 \bar{w}(0, z, t)$.

Detailed validation of the jet and plume simulations was performed in previous work $[35,36]$ for simulations at identical $\operatorname{Re}_{0}$. The results presented below are for a larger domain and are obtained with even higher resolutions. A detailed validation will thus not be repeated here; agreement with existing data will be pointed out in the text and, where appropriate, included in the figures. 
TABLE II. Asymptotic far-field solutions of jets and plumes including turbulence and pressure effects. In the expressions above, $M_{0}$ and $F_{0}$ are the mean specific momentum and buoyancy fluxes far away from the source.

\begin{tabular}{lcc}
\hline \hline Quantity & Jet & Plume \\
\hline$\Gamma$ & 0 & 1 \\
Ri & 0 & $8 \alpha_{p} \beta_{g} / 5$ \\
$r_{m}$ & $2 \alpha_{j} z$ & $\frac{6}{5} \alpha_{p} z$ \\
$w_{m}$ & $\frac{M_{0}^{1 / 2}}{2 \alpha_{j}} z^{-1}$ & $\frac{5}{6 \alpha_{p}}\left(\frac{9}{10} \frac{\alpha_{p}}{\theta_{m} \beta_{g}} F_{0}\right)^{1 / 3} z^{-1 / 3}$ \\
$b_{m}$ & $\frac{F_{0}}{2 \alpha_{j} \theta_{m}} M_{0}^{-1 / 2} z^{-1}$ & $\frac{5 F_{0}}{6 \alpha_{p} \theta_{m}}\left(\frac{9}{10} \frac{\alpha_{p}}{\beta_{g} \theta_{m}} F_{0}\right)^{-1 / 3} z^{-5 / 3}$ \\
\hline \hline
\end{tabular}

\section{RESULTS}

\section{A. Integral flow statistics}

From an integral perspective, the plume dynamics are fully determined by the evolution of the characteristic radius $r_{m}$, velocity $w_{m}$, and buoyancy $b_{m}$. For the limiting cases of a pure jet $(\Gamma=0)$ and of a pure plume $(\Gamma=1)$, the scaling of these parameters with the distance from the source takes the form of a power law, which can be derived from the plume equations [4]. Recently [26], these solutions were extended to account for turbulence and pressure effects via the profile coefficient $\beta_{g}$ and for differences in the widths of velocity and buoyancy profiles via the coefficient $\theta_{m}$ (Table II). The profile coefficients $\beta_{g}$ and $\theta_{m}$ will be defined rigorously in Sec. III C. The streamwise evolution of $r_{m}$ is shown in Fig. 1(a), confirming the almost identical linear spreading rate for the three simulations considered. Figures 1(b)-1(d) show that the jet and plume both exhibit the expected power-law scaling. The forced plume transitions from a near-field jetlike scaling to a far-field plumelike scaling.

As visible in Fig. 1(a), the outflow boundary condition appears to affect the statistics in the upper part of the domain. This is caused by subtle modification of the mean flow near the outflow boundary, presumably because of slight pressure gradients [34]. These small disturbances affect the integral quantities $Q, M$, and $F$ via the thresholding technique (which is based on $\bar{w}$; see Sec. II). Hereafter, all considerations on the dynamics of the flow will therefore be based on the analysis of the flow statistics for $z / r_{0}<50$.

For the two limiting cases $\mathrm{J}$ and $\mathrm{P}$, the plume radius $r_{m}(z)$ is fitted in the far field $\left(20<z / r_{m}<50\right)$ to the analytical solutions $r_{m}=a \alpha\left(z-z_{v}\right)$, where $z_{v}$ is the virtual origin [29] and $a=2$ for jets and $a=6 / 5$ for plumes (see Table II). We obtain $\alpha_{j}=0.067$ and $\alpha_{p}=0.105$, values that agree well with the literature and provide evidence of enhanced dilution within a plume compared to a jet.

A flux balance parameter $\Gamma(z)$ that takes into account turbulence, pressure effects, and differences in profile widths is defined as [26]

$$
\Gamma=\frac{5 F Q^{2}}{8 \alpha_{p} \beta_{g} \theta_{m} M^{5 / 2}}
$$

and its variation with height is shown for the three simulations in Fig. 2(a). For simulation $J, \Gamma$ is identically zero for all values of $z$. For simulation $\mathrm{P}, \Gamma \approx 1$ except for a rapid variation in the very near field $z / r_{0}<5$. It is worth noting that for simulation $\mathrm{P}$, the turning points of $\Gamma$ in the near field are not compatible with classical solutions of the plume equations [15] and have to be attributed to the near-field variations of the profile coefficients (Sec. III C). For forced plume simulation F, $\Gamma$ evolves approximately linearly towards its equilibrium state $\Gamma=1$, a condition that is however not attained at the upper limit of the simulated domain. 
(a)

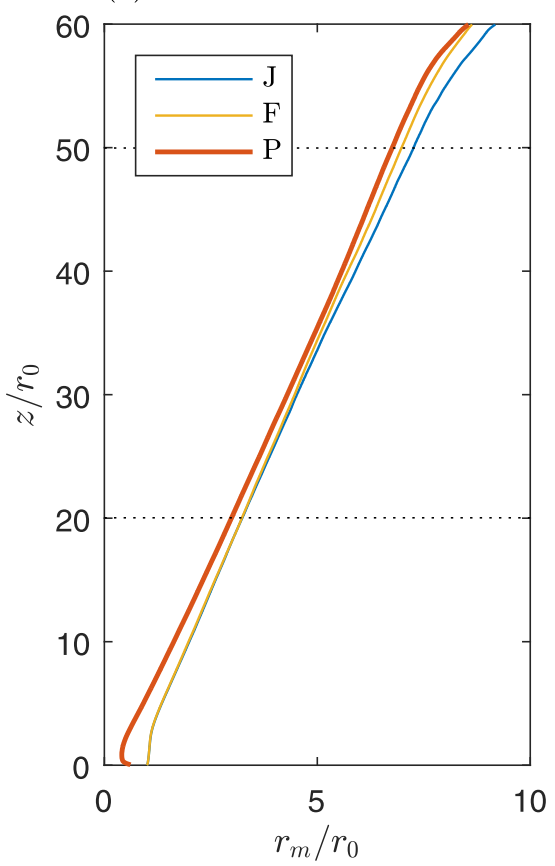

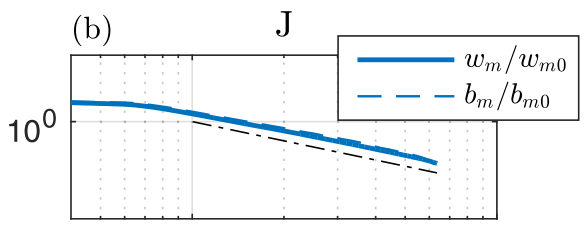

(c)

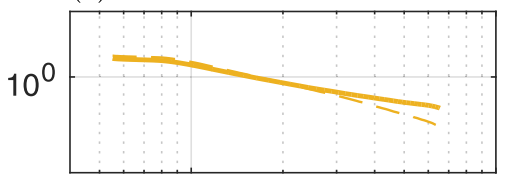

(d)

P

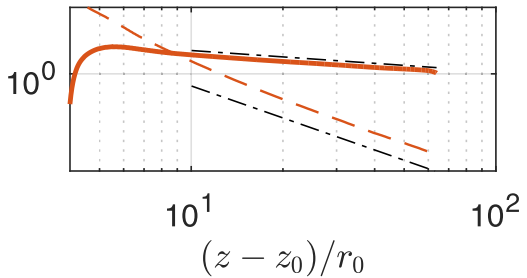

FIG. 1. Variation of the characteristic plume quantities with height $z$ for simulations J, F, and P: (a) $r_{m}(z)$, (b) $b_{m}$ and $w_{m}$ for $\mathrm{J}$ release, (c) $b_{m}$ and $w_{m}$ for $\mathrm{F}$ release, and (d) $b_{m}$ and $w_{m}$ for $\mathrm{P}$ release. Dash-dotted lines in (b)-(d) show asymptotic power-law scaling (Table II).

(a)

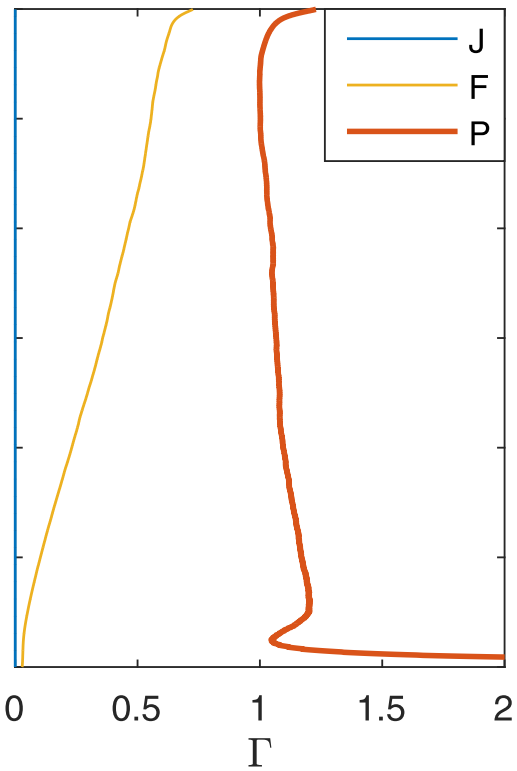

(b)

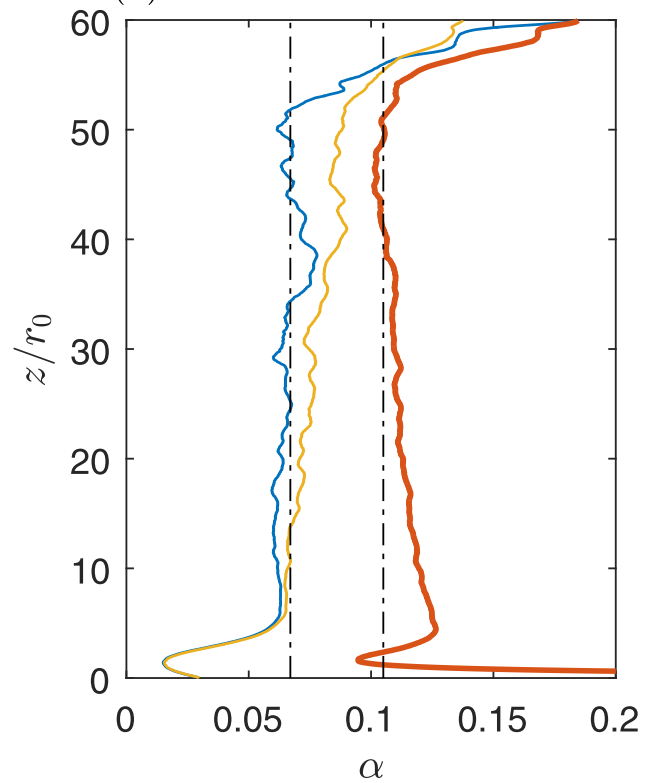

FIG. 2. Vertical evolution for the simulations J, F, and P of (a) the flux balance parameter $\Gamma$ and (b) the entrainment coefficient $\alpha$ computed from (6). 


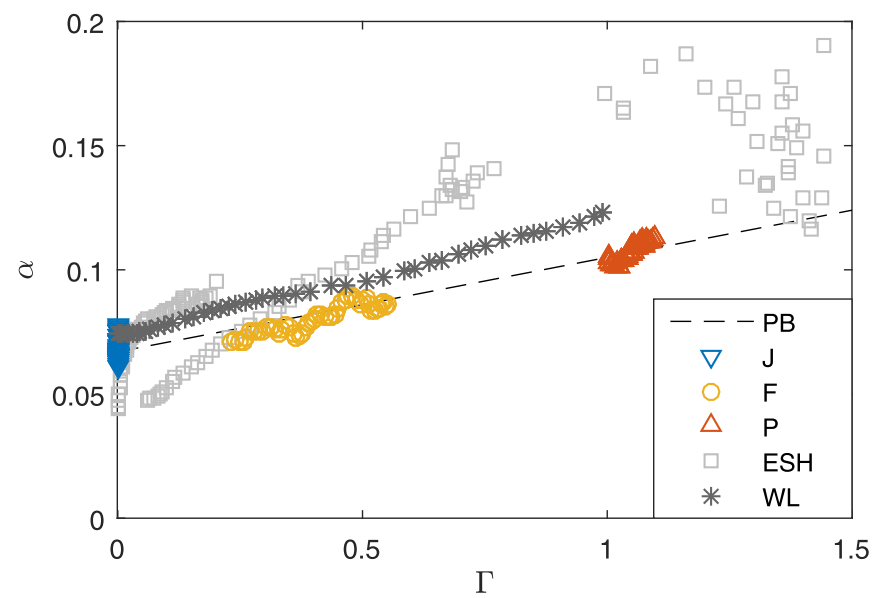

FIG. 3. Entrainment coefficient $\alpha$ as a function of $\Gamma$ over the interval $20<z / r_{0}<50$ for simulations J, F, and P, confirming the good agreement with the PB [3] entrainment model. The WL [9] and ESH [25] data are also shown.

The variation of the entrainment coefficient $\alpha$ with the vertical coordinate $z$, as determined from (6), is plotted in Fig. 2(b). Here $Q$ was filtered to smooth out occasional small step changes in its value caused by the thresholding, which would otherwise result in unphysical spikes in $d Q / d \zeta$ and $\alpha(z)$. The values of $\alpha_{j}$ and $\alpha_{p}$ (Table I) inferred from $r_{m}$ are displayed with the dash-dotted lines and are in good agreement with the far-field values for the jet and the plume, respectively. The entrainment in the pure jet shows a high variability in the near field but rapidly attains the constant value $\alpha_{j}$, within no more than five source radii. The entrainment coefficient for simulations $\mathbf{J}$ and $\mathrm{F}$ are almost the same in the near field. However, with increasing distance from the source, the entrainment coefficient in the forced plume simulation $\mathrm{F}$ shows a clear increasing trend. For the pure plume, the entrainment coefficient is very large in the near field $\left(z / r_{0}<5\right)$ and then attains an approximately constant value, which is in close agreement with the far-field estimate $\alpha_{p}=0.105$ obtained from $r_{m}$. These results are in agreement with previous experimental investigations [9,25] and show a clear tendency of the entrainment coefficient to increase with increasing $\Gamma$.

By plotting the computed values of $\alpha$ as a function of $\Gamma$, it is possible to test directly the appropriateness of the Priestley-Ball (PB) [3] entrainment model (12) (see Fig. 3). Shown in the same plot is the experimental data from Wang and Law [9] and the recent measurements from Ezzamel et al. [25]. The latter has been reprocessed in the Appendix to better represent the coflow in the ambient, which significantly influences the entrainment statistics. The new ambient-flow correction shows much better agreement between the volume-flux-based estimate of $\alpha$ and that obtained from the entrainment relation, although the data do not display the constant value of $\alpha$ that one would expect from self-similarity in the far field for the jet and plume experiments.

As is evident from Fig. 3, all data sets show a dependence on $\Gamma$. The current DNS data set and the Wang-Law (WL) data convincingly demonstrate the linear dependence on $\Gamma$ of the Priestley-Ball entrainment model (12) for unstratified environments in the self-similar regime. However, the figure also exposes the variability in what may be regarded as the limiting (or end member) entrainment coefficients; the values one would choose for $\alpha_{j}$ and $\alpha_{p}$ in Eq. (12) would be slightly different for the WL and current data sets. The dashed line shows the PB entrainment model using the values of $\alpha_{j}$ and $\alpha_{p}$ presented in Table I and good agreement with the DNS data can be observed. The Ezzamel-Salizzoni-Hunt (ESH) data confirm the appropriateness of the PB model qualitatively, but despite the ambient-flow correction (Appendix) the data remain noisy. The linear dependence of $\alpha$ on $\Gamma$ implies that $\delta_{m}$ is practically identical in jets and plumes, as argued in the Introduction. The entrainment coefficient will be decomposed into its various parts in Sec. III D. 


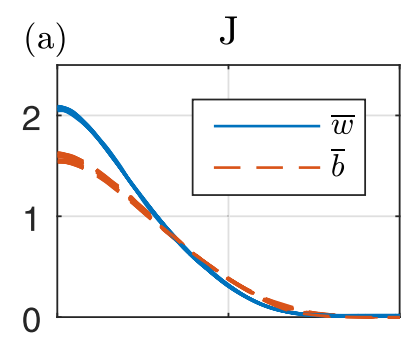

(d)

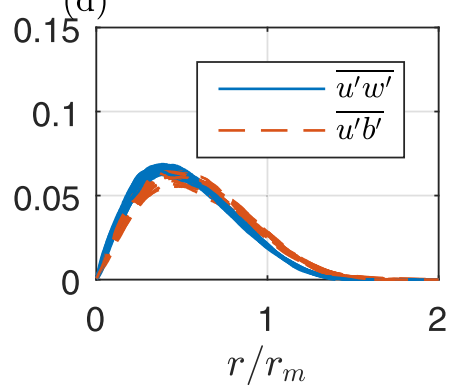

(b)

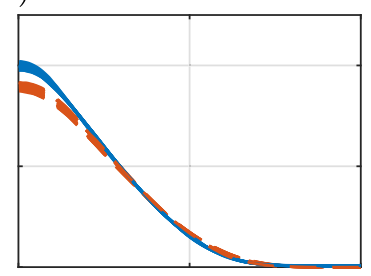

(e)

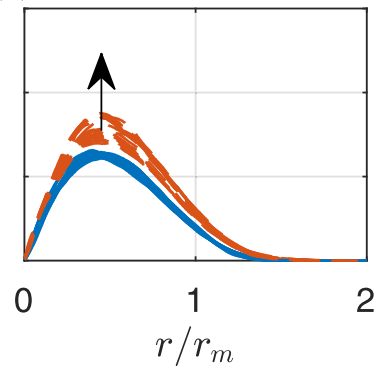

(c)

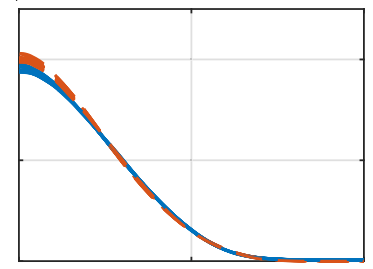

(f)

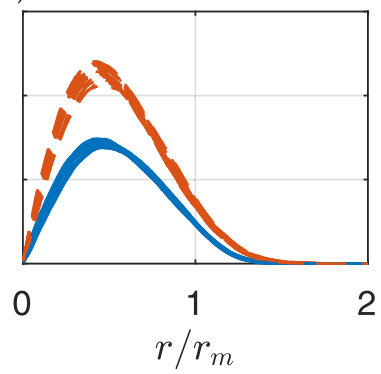

FIG. 4. Self-similarity profiles of $\bar{w}, \bar{b}, \overline{u^{\prime} w^{\prime}}$, and $\overline{u^{\prime} b^{\prime}}$ over the interval $20<z / r_{0}<50$ for (a) and (d) jet simulation, (b) and (e) forced plume simulation, and (c) and (f) pure plume simulation.

\section{B. Self-similarity}

Shown in Fig. 4 are the mean velocity $\bar{w}$, buoyancy $\bar{b}$, radial turbulent momentum flux $\overline{u^{\prime} w^{\prime}}$, and turbulent buoyancy flux $\overline{u^{\prime} b^{\prime}}$ over the vertical interval $20<z / r_{0}<50$. As is customary, all variables are presented in dimensionless form, normalized by the local value of $r_{m}, b_{m}$, and $w_{m}$. In line with our expectations, for all three simulations the mean vertical velocity $\bar{w}$ collapses onto a single profile that closely resembles a Gaussian profile.

The radial profiles of mean buoyancy $\bar{b}$ also exhibit a clear Gaussian-like dependence on the radial coordinate. However, the centerline values and spread differ for the three simulations. Profiles for velocity and buoyancy almost coincide for plumes [Fig. 4(c)], whereas for the forced plume and the jet, the buoyancy profiles have a slightly larger spread (as further quantified by the profile coefficient $\theta_{m}$ associated with mean scalar transport; see Sec. IIIC). As the integral under the dimensionless curves is unity by construction, a wider profile will reduce the centerline value of $\bar{b} / b_{m}$, particularly since small changes far from the centerline contribute significantly to the integral due to the conical geometry.

The profile of the turbulent radial momentum flux $\overline{u^{\prime} w^{\prime}}$ is practically identical for the jet, forced plume, and pure plume [Figs. 4(d)-4(f)], which is consistent with the notion of the profile coefficient associated with the production of turbulence kinetic energy $\delta_{m}$ being insensitive to $\Gamma$. However, the normalized radial turbulent buoyancy flux shows large variations in amplitude. For the jet simulations, the profiles of $\overline{u^{\prime} w^{\prime}}$ and $\overline{u^{\prime} b^{\prime}}$ are practically identical. For the plume simulation, $\overline{u^{\prime} b^{\prime}}$ is about $60 \%$ larger in amplitude than $\overline{u^{\prime} w^{\prime}}$. The profile of $\overline{u^{\prime} b^{\prime}}$ for the forced plume transitions smoothly from the jet profile to the plume profile as $\Gamma$ tends to unity, as indicated by the arrow in Fig. 4(e); this is in contrast to Fig. 4(f), where no systematic variation with height is present.

The normalized mean radial velocity $\bar{u}$ is shown in Fig. 5(a). Contrary to the mean vertical velocity $\bar{w}$ profiles, the shape of $\bar{u}$ differs significantly between the jet, forced plume, and pure plume. For the jet, $\bar{u}$ increases from a value of zero (imposed by the radial symmetry of the flow), reaches a peak at $r / r_{m} \approx 0.5$, then decreases, becomes negative with a minimum at $r / r_{m} \approx 1.4$, and then decays approximately inversely proportional to the radius due to the fact that the flow varies very slowly 
(a)

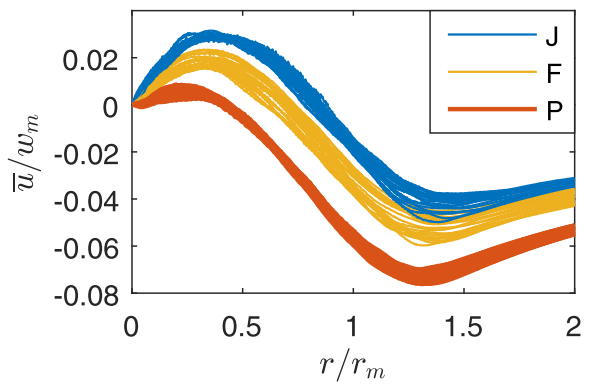

(b)

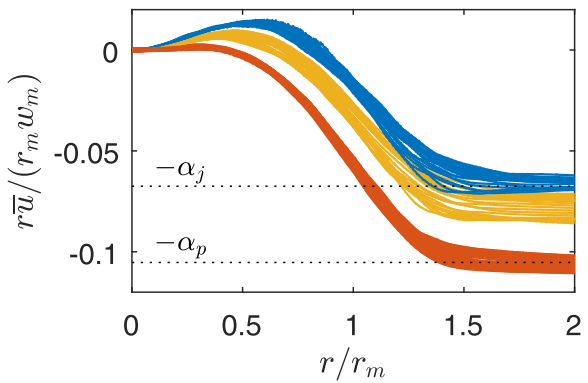

FIG. 5. (a) Self-similar profiles for mean radial velocity $\bar{u}$ and (b) normalized mean radial specific volume flux. The dotted lines indicates the values of $\alpha_{j}$ and $\alpha_{p}$ in Table I.

with $z$. For the plume, the maximum in $\bar{u}$ is significantly smaller, implying a reduction in the mean outward radial transport in a plume. The normalized specific radial volume flux $r \bar{u} / r_{m} w_{m}$, shown in Fig. 5(b) for all three simulations, tends to a constant value outside the plume for $r / r_{m}>1.5$. By rearranging Eq. (5), it clear that the constant value is equal to the entrainment coefficient $\alpha$. The
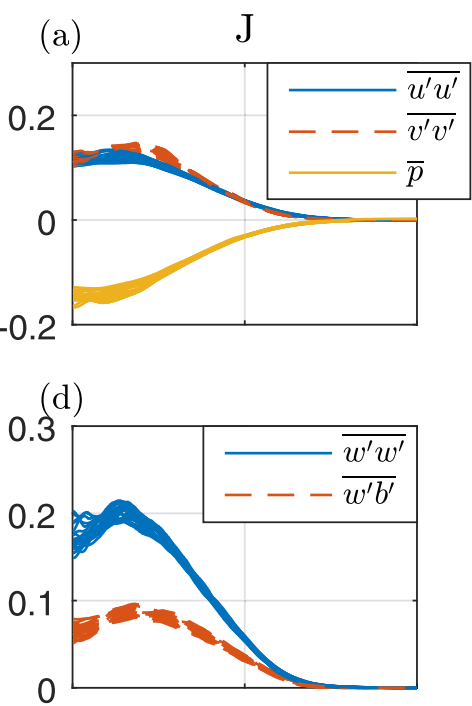

$(\mathrm{g})$

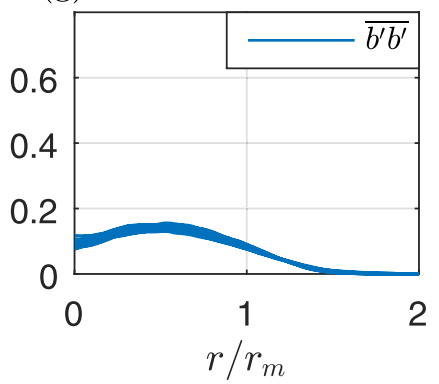

(b)

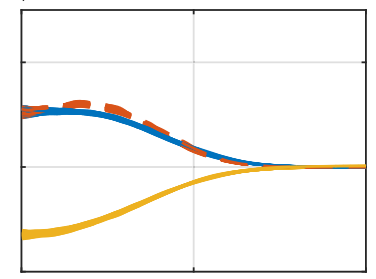

(e)

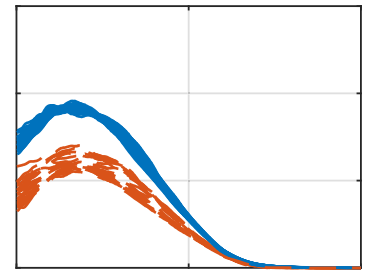

(h)

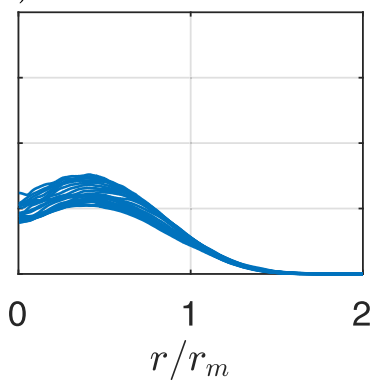

(c)

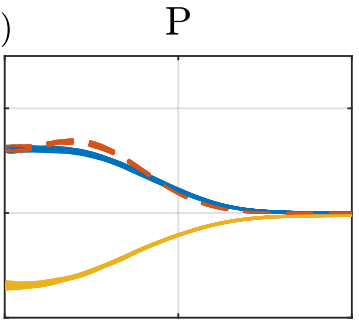

(f)

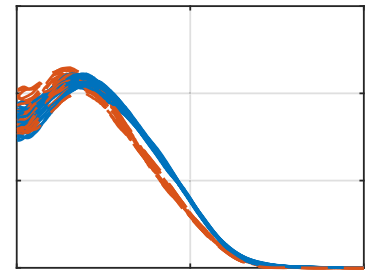

(i)

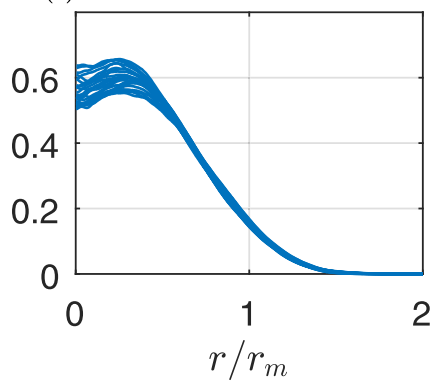

FIG. 6. Self-similarity profiles of second-order quantities and pressure. All quantities are normalized. 


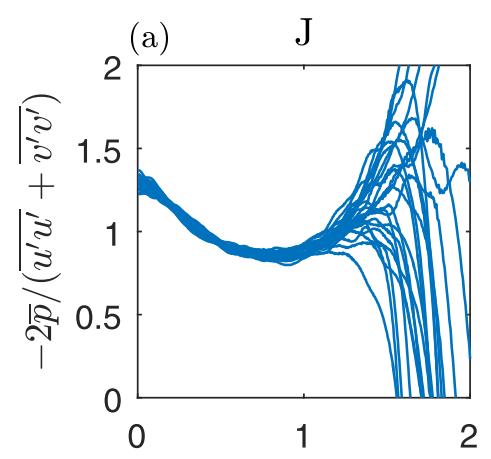

(d)

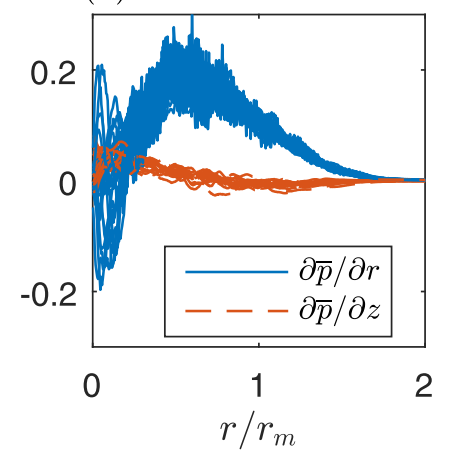

(b)

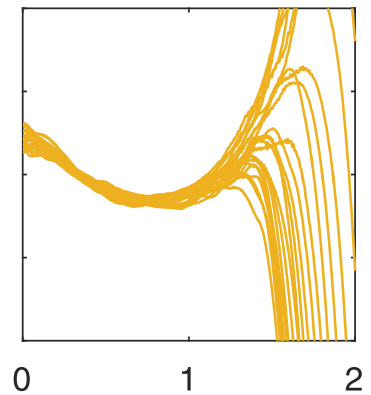

(e)

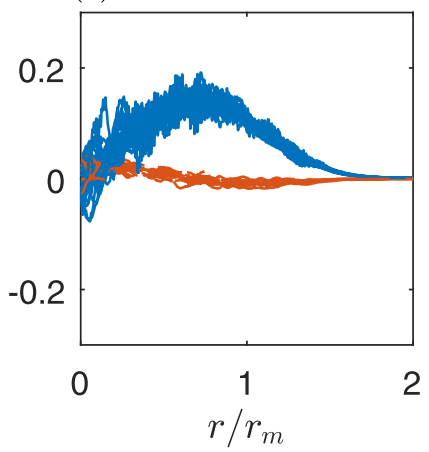

(c) $\quad \mathrm{P}$

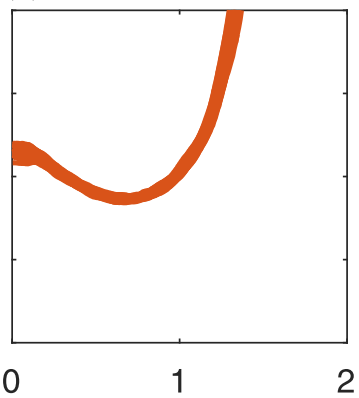

(f)

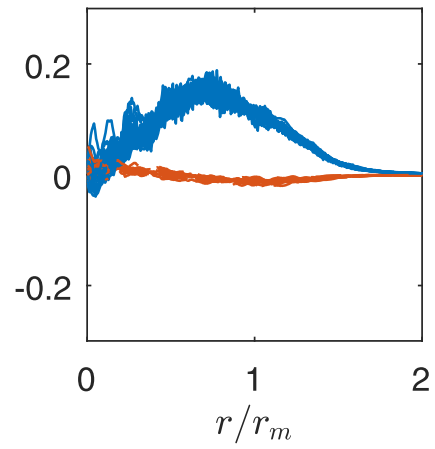

FIG. 7. (a)-(c) Ratio of mean pressure $\bar{p}$ and $-\left(\overline{u^{\prime} u^{\prime}}+\overline{v^{\prime} v^{\prime}}\right) / 2$ and (d)-(f) horizontal and vertical pressure gradients for (a) and (d) simulation J, (b) and (e) simulation F, and (c) and (f) simulation P.

dashed lines in Fig. 5(b) are the values of $\alpha$ in Table I; excellent agreement is shown with the values deduced from $r_{m}$.

The turbulent components $\overline{u^{\prime} u^{\prime}}$ and $\overline{v^{\prime} v^{\prime}}$, shown as a function of $r / r_{m}$ in Figs. 6(a)-6(c), are selfsimilar and practically identical. Furthermore, their dependence on $\Gamma$ is negligible, providing further confirmation that the turbulence inside plumes and jets is similar, at least in terms of the second-order statistics. The mean pressure $\bar{p}$ is extremely difficult to measure in laboratory experiments and is usually approximated by $[9,25,37] \bar{p} \approx-\left(\overline{u^{\prime} u^{\prime}}+\overline{v^{\prime} v^{\prime}}\right) / 2$. The quantity $\bar{p}$ is readily available in DNS and it is clear from Figs. 6(a)-6(c) that it correlates well with $-\left(\overline{u^{\prime} u^{\prime}}+\overline{v^{\prime} v^{\prime}}\right) / 2$, although upon closer inspection (Fig. 7) it becomes evident that $-\left(\overline{u^{\prime} u^{\prime}}+\overline{v^{\prime} v^{\prime}}\right) / 2$ underestimates $\bar{p}$ by $30 \%$ in the core of the flow, while it overestimates $\bar{p}$ by about $10 \%$ near $r / r_{m}=1$. Thus, the DNS data demonstrate that $\bar{p}=-\left(\overline{u^{\prime} u^{\prime}}+\overline{v^{\prime} v^{\prime}}\right) / 2$ within, say, $20 \%$ (see [37] for a detailed explanation of the various sources of error). Like the gradient of all quantities in a slender turbulent boundary layer, the gradient of pressure in the radial direction is expected to be larger than in the vertical direction by a factor proportional to the spreading rate of the flow. The DNS data confirm that this is the case [Figs. 7(d)-7(f)].

Figures 6(d)-6(f) show the streamwise turbulent momentum and buoyancy flux. While the vertical turbulent momentum flux is more or less identical for cases J, F, and P, the buoyancy profile differs significantly between the three subplots. Clearly, an increase in the value of $\Gamma$ increases the vertical turbulent buoyancy flux, as well as the radial buoyancy flux [Figs. 4(d)-4(f)]. A similar trend is observable in the turbulence buoyancy variance [Figs. 6(g)-6(i)]. Note that given a sufficient vertical extent of the domain, we expect both $\overline{w^{\prime} b^{\prime}}$ and $\overline{b^{\prime} b^{\prime}}$ for simulation $\mathrm{F}$ to increase to levels observed in simulation $\mathrm{P}$. 
(a)

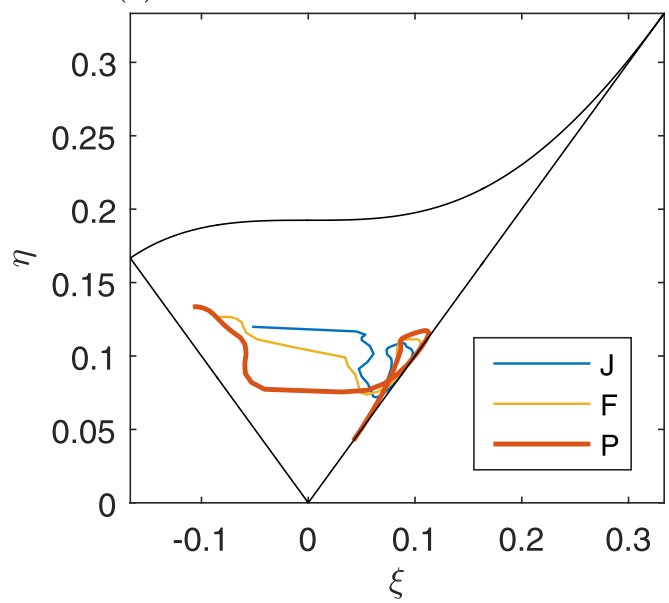

(b)
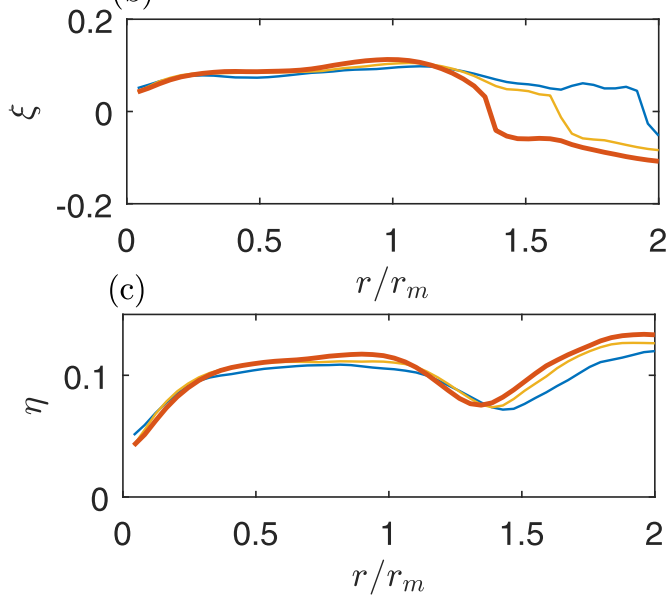

FIG. 8. Invariants of the anisotropy tensor (16) for the jet, forced plume, and plume. (a) Plot in $\xi-\eta$ space, together with the Lumley triangle. (b) Dependence of $\xi$ on $r / r_{m}$. (c) Dependence of $\eta$ on $r / r_{m}$.

To provide further evidence of the similarity of the turbulence statistics in plumes and jets it is instructive to calculate the invariants of the anisotropy tensor [38]

$$
b_{i j}=\frac{\overline{u_{i}^{\prime} u_{j}^{\prime}}}{2 e}-\frac{1}{3} \delta_{i j},
$$

where $e=\frac{1}{2} \overline{u_{i}^{\prime} u_{i}^{\prime}}$ is the turbulence kinetic energy and $\delta_{i j}$ is the Kronecker delta. As the turbulence is incompressible, one invariant of $\mathbf{b}$ is zero and the other two, denoted $\xi$ and $\eta$, are defined via $\operatorname{Tr}\left(\mathbf{b}^{2}\right) \equiv 6 \xi^{2}$ and $\operatorname{Tr}\left(\mathbf{b}^{3}\right) \equiv 6 \eta^{3}$, where $\operatorname{Tr}$ denotes the tensor trace. The invariants of $\mathbf{b}$ cannot take any value; realizable flows are confined to a region of the $\xi-\eta$ space commonly known as the Lumley triangle [38].

The invariants are calculated as follows. The second-order statistics shown in Figs. 4 and 6 are averaged over the range $20<z / r_{m}<50$, after which $\xi$ and $\eta$ are calculated as a function of $r / r_{m}$. Figures 8(b) and 8(c) show, respectively, the profiles of invariants $\eta$ and $\xi$ as a function of $r / r_{m}$. It is evident that the profiles for $\mathrm{J}, \mathrm{F}$, and $\mathrm{P}$ are nearly indistinguishable for $r / r_{m}<1.5$, providing further evidence that turbulence in jets and plumes is similar. In the $\xi-\eta$ plane [Fig. 8(a)], the data are close to the $\xi=\eta$ line, which is indicative of axisymmetric turbulence with one large eigenvalue, i.e., rodlike turbulence. Interestingly, at the edge of the jet or plume, $\xi$ changes very rapidly from positive to negative. For plumes, the crossover appears to happen closer to the centerline than for the jet. Thus, near the plume edge, the average picture of the turbulence resembles axisymmetric turbulence with one small eigenvalue, i.e., disklike turbulence. These observations are in agreement with the laboratory experiments of Hussein et al. [37], which were presented in terms of the $(\xi, \eta)$ invariants in [39].

Consideration of the vertical gradient $\partial \bar{w} / \partial z$ provides a possible explanation for why the point at which turbulence changes from being dominated by one component (the core region) to two components (the edge of the flow) differs in jets compared with plumes. Noting that $w_{m} \sim z^{-1}$ in jets, whereas $w_{m} \sim z^{-1 / 3}$ in plumes, the point at which $\partial \bar{w} / \partial z=0$ occurs at larger values of $r / r_{m}$ in jets than it does in plumes. Likening the flow with a diverging (core region, $\partial \bar{w} / \partial z<0$ ) or converging (edge region, $\partial \bar{w} / \partial z>0$ ) nozzle, one would therefore expect the point of transition between one-component and two-component regimes, respectively, to be affected by differences in the point at which $\partial \bar{w} / \partial z$ changes sign. 


\section{Profile coefficients}

Profile coefficients encapsulate integrated information about the mean and turbulent fluxes of momentum, buoyancy, mean kinetic, and turbulence production. In classical integral descriptions of the plume equations [4], the profile coefficients are generally assumed to be either unity or zero. However, preserving information about profile shapes is crucial in the description of unsteady jets and plumes $[35,36,40]$ and is also the key to decomposing entrainment into its various processes. The profile coefficients for the fluxes of momentum $\beta$, buoyancy $\theta$, and mean kinetic energy $\gamma$, as well as the dimensionless turbulence production $\delta$ are given by, respectively,

$$
\begin{aligned}
& \beta_{m} \equiv \frac{M}{w_{m}^{2} r_{m}^{2}} \equiv 1 \\
& \beta_{f} \equiv \frac{2}{w_{m}^{2} r_{m}^{2}} \int_{0}^{\infty} \overline{w^{\prime 2}} r d r, \quad \beta_{p} \equiv \frac{2}{w_{m}^{2} r_{m}^{2}} \int_{0}^{\infty} \bar{p} r d r, \\
& \gamma_{m} \equiv \frac{2}{w_{m}^{3} r_{m}^{2}} \int_{0}^{\infty} \bar{w}^{3} r d r \\
& \gamma_{f} \equiv \frac{4}{w_{m}^{3} r_{m}^{2}} \int_{0}^{\infty} \bar{w} \overline{w^{\prime 2}} r d r, \quad \gamma_{p} \equiv \frac{4}{w_{m}^{3} r_{m}^{2}} \int_{0}^{\infty} \overline{w p} r d r, \\
& \delta_{m} \equiv \frac{4}{w_{m}^{3} r_{m}} \int_{0}^{\infty} \overline{w^{\prime} u^{\prime}} \frac{\partial \bar{w}}{\partial r} r d r \\
& \delta_{f} \equiv \frac{4}{w_{m}^{3} r_{m}} \int_{0}^{\infty} \overline{w^{\prime 2}} \frac{\partial \bar{w}}{\partial z} r d r, \quad \delta_{p} \equiv \frac{4}{w_{m}^{3} r_{m}} \int_{0}^{\infty} \bar{p} \frac{\partial \bar{w}}{\partial z} r d r, \\
& \theta_{m} \equiv \frac{F}{w_{m} b_{m} r_{m}^{2}} \\
& \theta_{f} \equiv \frac{2}{w_{m} b_{m} r_{m}^{2}} \int_{0}^{\infty} \overline{w^{\prime} b^{\prime}} r d r
\end{aligned}
$$

The total momentum flux is given by $\beta_{g} M$, where $\beta_{g}=\beta_{m}+\beta_{f}+\beta_{p}$. Similarly, $\theta_{g}$ is associated with the total buoyancy flux, $\gamma_{g}$ with the total energy flux, and $\delta_{g}$ with the total turbulence production (including pressure redistribution). Profile coefficients $\beta$ and $\theta$ show up naturally upon radial integration of the Reynolds-averaged volume, vertical momentum, and buoyancy equations of a high-Reynolds-number flow in a neutral environment [26]

$$
\begin{aligned}
\frac{1}{Q} \frac{d Q}{d \zeta} & =2 \alpha \\
\frac{1}{M} \frac{d}{d \zeta}\left(\beta_{g} M\right) & =\mathrm{Ri} \\
\frac{1}{F} \frac{d}{d \zeta}\left(\frac{\theta_{g}}{\theta_{m}} F\right) & =0
\end{aligned}
$$

These equations reduce to the classical plume equations [4] on setting $\beta_{g}=1$ and $\theta_{g}=\theta_{m}=1$. Furthermore, we note that $\mathrm{Ri}=0$ by definition for the jet, implying that the evolution of $F$ and $M$ are uncoupled (and that $F$ in that case corresponds to a passive scalar flux). Similarly, $\gamma$ and $\delta$ emerge naturally from integration of the mean kinetic energy equation

$$
\frac{Q}{M^{2}} \frac{d}{d \zeta}\left(\gamma_{g} \frac{M^{2}}{Q}\right)=\delta_{g}+2 \theta_{m} \mathrm{Ri}
$$

Figure 9 shows the profile coefficients as a function of $z$. The coefficients associated with the mean flow, $\beta_{m}, \gamma_{m}, \delta_{m}$, and $\theta_{m}$, are shown in Figs. 9(a)-9(c). There are large variations in the profile coefficients in the near field, which are due to changes in the velocity and buoyancy profiles as the jet or plume develops; indeed, the largest changes occur over a small region $z / r_{0}<5$, for the plume even closer to the source $\left(z / r_{0}<3\right)$. However, for larger $z / r_{0}$ the coefficients become constant, which is consistent with self-similarity.

The average values of the profile coefficients over the interval $20<z / r_{0}<50$ are presented in Table III. The dimensionless buoyancy flux $\theta_{m}$ is less than unity for the jet, implying that the spread of the buoyancy field exceeds the spread of the velocity field. This can be shown by assuming a 


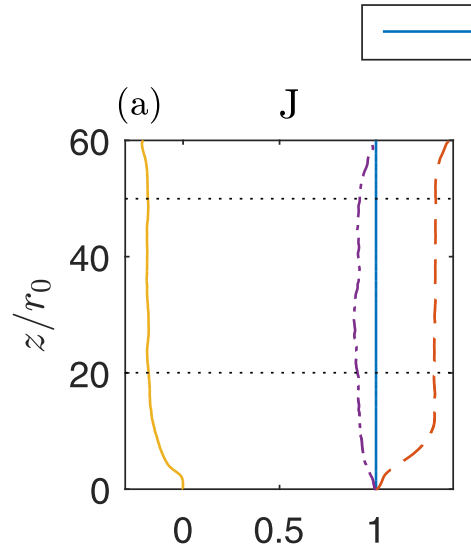

(d)

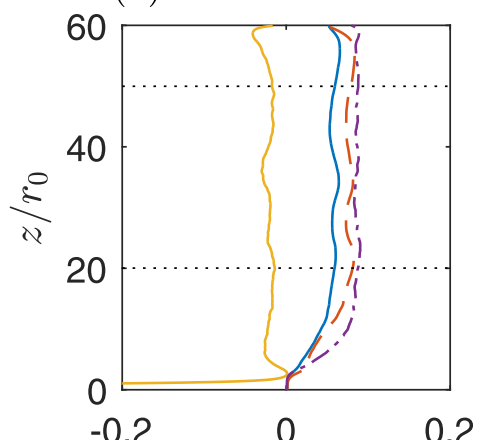

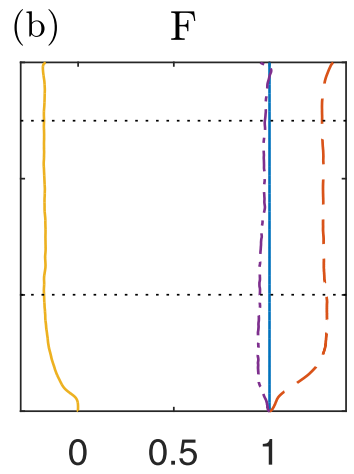

(e)

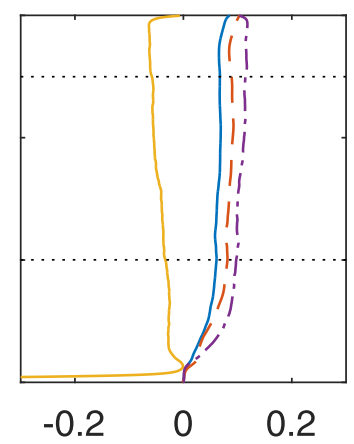

(c) $\quad \mathrm{P}$

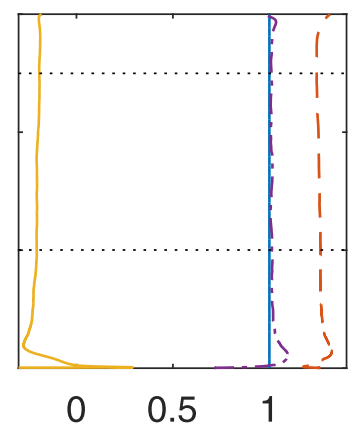

(f)

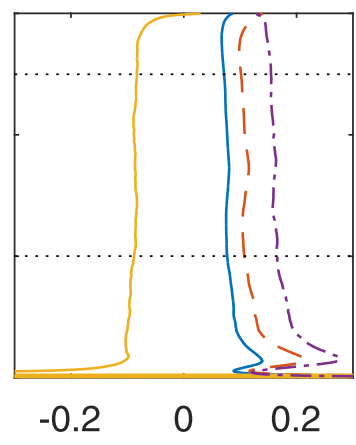

$$
-\beta_{g}-1---\left(\gamma_{g}-\gamma_{m}\right) / \gamma_{m}-\left(\delta_{g}-\delta_{m}\right) / \delta_{m}-\cdot-\cdot-\left(\theta_{g}-\theta_{m}\right) / \theta_{m}
$$

FIG. 9. (a)-(c) Mean profile coefficients and (d)-(f) relative contribution of turbulence and pressure to the dimensionless coefficients for (a) and (d) simulation J, (b) and (e) simulation F, and (c) and (f) simulation P. The dashed lines indicate the averaging interval $20<z / r_{0}<50$ used for the profile coefficients displayed in Table III.

Gaussian form for the velocity and buoyancy profiles

$$
\bar{w}=2 w_{m} \exp \left(-2 \frac{r^{2}}{r_{m}^{2}}\right), \quad \bar{b}=2 \frac{b_{m}}{\varphi^{2}} \exp \left(-2 \frac{r^{2}}{\varphi^{2} r_{m}^{2}}\right),
$$

where $\varphi r_{m}$ is the characteristic width of the buoyancy profile and $\varphi$ is the ratio of the buoyancy to velocity radii. These profiles are consistent with the definitions $\beta_{m}=1$ and $B=b_{m} r_{m}^{2}$ and evaluation of the profile coefficient for the mean energy flux results in $\gamma_{m}=4 / 3$. The buoyancy flux is given by $F=2 \int_{0}^{\infty} \bar{w} \bar{b} r d r=\frac{2}{\varphi^{2}+1} w_{m} b_{m} r_{m}^{2}$. By substituting this expression into the definition of profile coefficient $\theta_{m}$ (17), it directly follows that

$$
\theta_{m}=\frac{2}{\varphi^{2}+1}
$$

For the plume, $\theta_{m} \approx 1$, implying that $\varphi \approx 1$ also. The value of $\theta_{m}$ for the forced plume tends to become closer to unity with increasing $z$. The dimensionless turbulence production $\delta_{m}$ shows differences of the order of $10 \%$ between the jet and the plume (see also Table III), which is too small to explain the observed differences in $\alpha$ (see Sec. III D). 
TABLE III. Average profile coefficients over the interval $20<$ $z / r_{0}<50$.

\begin{tabular}{lrrr}
\hline \hline Coefficient & \multicolumn{1}{c}{$\mathrm{J}$} & $\mathrm{F}$ & \multicolumn{1}{c}{$\mathrm{P}$} \\
\hline$\beta_{f}$ & 0.151 & 0.149 & 0.183 \\
$\beta_{u}$ & 0.095 & 0.088 & 0.106 \\
$\beta_{v}$ & 0.102 & 0.095 & 0.110 \\
$\beta_{p}$ & -0.093 & -0.084 & -0.107 \\
$\beta_{g}$ & 1.058 & 1.065 & 1.076 \\
$\gamma_{m}$ & 1.306 & 1.282 & 1.256 \\
$\gamma_{f}$ & 0.276 & 0.267 & 0.319 \\
$\gamma_{p}$ & -0.175 & -0.156 & -0.183 \\
$\gamma_{g}$ & 1.406 & 1.393 & 1.391 \\
$\delta_{m}$ & -0.184 & -0.175 & -0.201 \\
$\delta_{f}$ & 0.006 & 0.016 & 0.038 \\
$\delta_{p}$ & -0.002 & -0.008 & -0.021 \\
$\delta_{g}$ & -0.180 & -0.167 & -0.184 \\
$\theta_{m}$ & 0.901 & 0.964 & 1.011 \\
$\theta_{f}$ & 0.078 & 0.103 & 0.162 \\
$\theta_{g}$ & 0.979 & 1.067 & 1.172 \\
\hline \hline
\end{tabular}

Figures 9(d)-9(f) show the relative contribution of turbulence and pressure terms to the total, which are neglected in classical plume theory. Gradual changes can be observed in the far field that are caused by the fact that the second-order statistics require a greater vertical distance to become fully self-similar than the first-order statistics. Indeed, Wang and Law [9] observed that full self-similarity of the turbulence statistics did not occur before $z / r_{0} \approx 100$, which is nearly twice the vertical extent of our domain. However, it is clear that, in general, the influence of turbulence and pressure is less than $10 \%$ of the mean value, which partially explains why plume theory provides such robust predictions for plume behavior. The largest deviations between mean and total are found in $\theta$, the dimensionless buoyancy flux, which for plumes is as high as $20 \%$, consistent with the literature [32,41]. Here we would like to point out that $\theta_{f}$ is a source of systematic error in laboratory experiments where the (total) buoyancy flux is usually determined a priori [(nozzle volume flux $) \times($ buoyancy)]. However, plume theory only considers means and the mean buoyancy flux is about $20 \%$ less than the total buoyancy flux. Indeed, we find good agreement of the DNS data with the classical solutions of plume theory only by explicitly calculating the mean buoyancy flux.

\section{Decomposing the entrainment coefficient}

As shown by van Reeuwijk and Craske [26], taking (6) as a definition of $\alpha$ and using (19) and (18b), $\alpha$ can be decomposed as

$$
\alpha=\underbrace{-\frac{\delta_{g}}{2 \gamma_{g}}}_{\alpha_{\text {prod }}}+\underbrace{\left(\frac{1}{\beta_{g}}-\frac{\theta_{m}}{\gamma_{g}}\right) \operatorname{Ri}}_{\alpha_{\mathrm{Ri}}}+\underbrace{\frac{d}{d \zeta}\left(\log \frac{\gamma_{g}^{1 / 2}}{\beta_{g}}\right)}_{\alpha_{\text {shape }}} .
$$

The entrainment relation (22) quantifies the contribution to $\alpha$ of turbulence production $\alpha_{\text {prod }}$, mean buoyancy $\alpha_{\mathrm{Ri}}$, and changes in profile shape $\alpha_{\text {shape }}$. The vertical evolution of the individual contributions to $\alpha$, the direct estimate of $\alpha$ using (6), and the estimate of $\alpha$ using $r_{m}$ (Table I) are plotted in Fig. 10. The three estimates of $\alpha$ are in good agreement with each other, demonstrating the consistency of the data with the underlying integral equations. The analysis of data from the plume literature carried out by van Reeuwijk and Craske [26] highlighted that $\delta_{m}$, and thus $\alpha_{\text {prod }}$, 
(a)

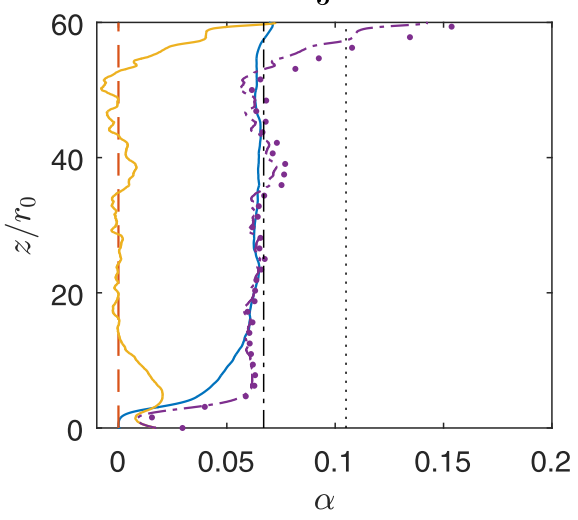

(c)

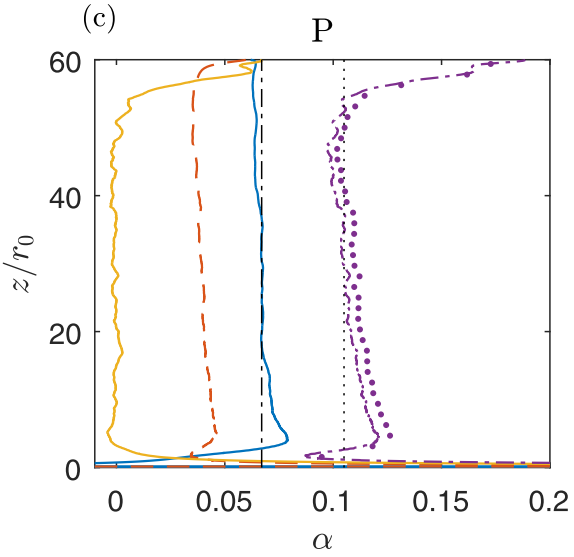

(b)
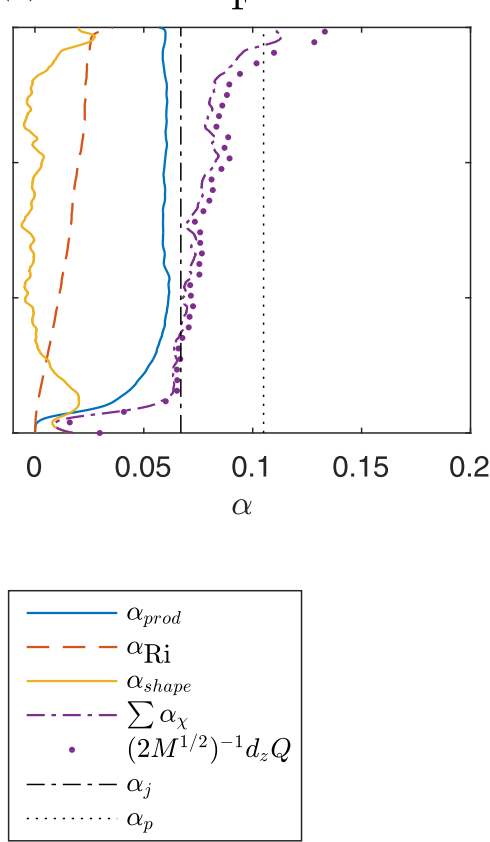

FIG. 10. Evolution of the contribution to entrainment due to turbulent kinetic energy production $\alpha_{\text {prod }}$, buoyancy $\alpha_{\mathrm{Ri}}$ and departure from self-similarity $\alpha_{\text {shape }}$, as a function of $z$. Note that in the legend, $\sum \alpha_{\chi}=$ $\alpha_{\text {prod }}+\alpha_{\text {Ri }}+\alpha_{\text {shape }}$.

was approximately identical in jets and plumes. This is convincingly confirmed in Fig. 10(c), as $\alpha_{\text {prod }}$ matches closely with the value of $\alpha_{j}$ inferred from the jet data. For the forced plume, $\alpha_{\text {prod }}$ is slightly lower than $\alpha_{j}$ but remains in good agreement. The mean-flow contribution of buoyancy to $\alpha$ is constant for simulation $\mathrm{P}$ and has a magnitude of $2 \alpha_{j} / 3$. For simulation $\mathrm{F}, \alpha_{\mathrm{Ri}}$ can be observed to increase with height.

The term $\alpha_{\text {shape }}$ will only be nonzero when the profiles of first- and second-order statistics change in shape, i.e., when the profiles are not self-similar. Non-self-similar behavior is dominant in the near field, where the flow transitions to turbulence and the mean profiles attain their Gaussian shapes. The near-field region, within which $\alpha_{\text {shape }}$ is different from zero, extends up to about 15 source diameters for the jet and the forced jet and only for about 5 source diameters for the plume.

Next we explore the concept of similarity drift, which pertains to a possible variation in $z$ of the ratio of buoyancy to velocity profile width $\varphi(z)$. The concept of similarity drift can be traced back to Kaminski et al. [17], who derived an entrainment relation that contains a term of the form

$$
\alpha_{e}=\cdots+\frac{1}{2} R \frac{d}{d z} \log A,
$$

where $R$ is a typical radius, $A=\gamma_{m} / \theta_{m}=\gamma_{m}\left(1+\varphi^{2}\right) / 2$, and $\alpha_{e}$ is an entrainment coefficient that is related [26], but not identical to $\alpha$ [ $\alpha_{e}$ uses nonstandard characteristic scales in Ref. [17], implying that the $\alpha_{\text {shape }}$ in the entrainment relation in terms of $\alpha$ (22) is independent of $\theta$ ]. Hence, (23) 

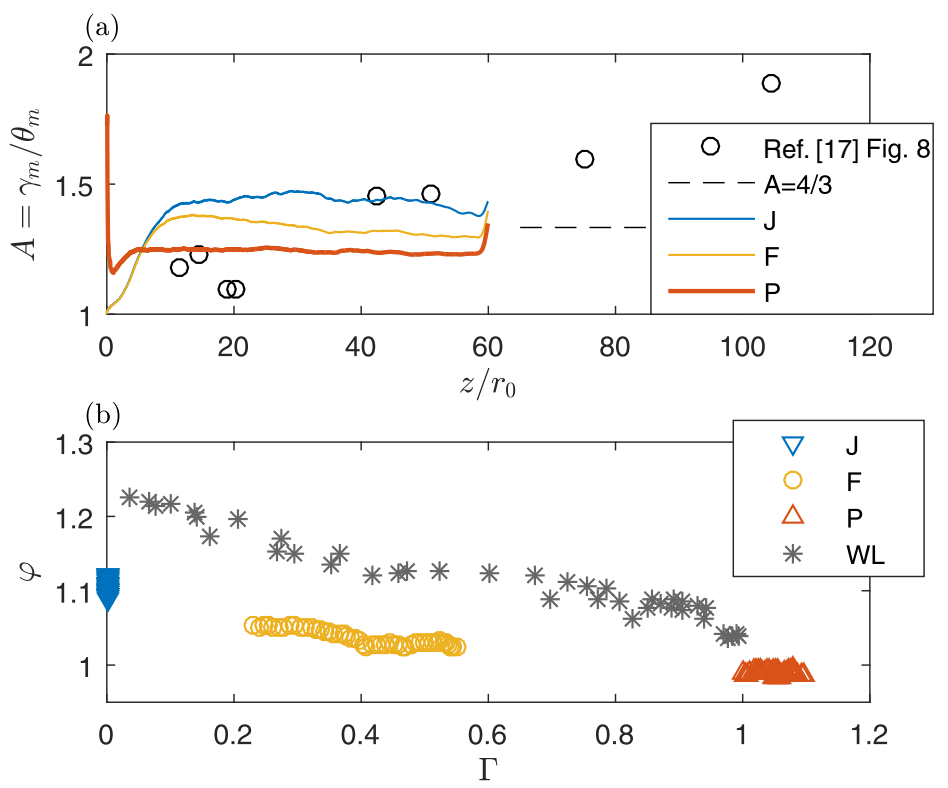

FIG. 11. Exploration of similarity drift for (a) $A=\gamma_{m} / \theta_{m}$ as a function of $z / r_{0}$ and (b) $\varphi$ as a function of $\Gamma$.

indicates that changes in $A$, e.g., because of a drift $\varphi=\varphi(z)$, will have a nonzero contribution to $\alpha_{e}$. In Ref. [17] the value $A$ was calculated for published data, which, despite significant scatter, showed an increasing trend of $A$ with the distance from the source.

Figure 11(a) shows the experimental data collected from Fig. 8 in Ref. [17] together with the DNS data set discussed in this article. Unlike the experimental data, the DNS data do not imply that $A$ varies as a function of $z$. Indeed, it is unclear what physical mechanism could be responsible for producing similarity drift. Full self-similarity of the process results from an asymptotically small dependence on the source conditions and ambient conditions that scale in the same way as the local behavior of the plume. We therefore suggest that the similarity drift observed in experiments is caused by the absence of an ideal undisturbed, unbounded ambient environment (including confinement effects), or a persistent dependence of the process on source conditions.

The DNS and WL data suggest a relation between $\varphi$ and $\Gamma$ [see Fig. 11(b)]. As for Fig. 3, the DNS and WL data show that $\varphi$ is a decreasing function of $\Gamma$, tending to $\varphi \approx 1$ at $\Gamma=1$. The $\Gamma$ dependence is more pronounced for the WL data than the DNS data, the reason for which is unclear.

\section{E. Turbulent transport}

The turbulent radial transport of streamwise momentum $\overline{u^{\prime} w^{\prime}}$ and buoyancy $\overline{u^{\prime} b^{\prime}}$ are crucial in determining the profile shape and entrainment behavior of jets and plumes. These quantities can be related to the mean fields using the gradient diffusion hypothesis, i.e.,

$$
\overline{u^{\prime} w^{\prime}}=-v_{T} \frac{\partial \bar{w}}{\partial r}, \quad \overline{u^{\prime} b^{\prime}}=-D_{T} \frac{\partial \bar{b}}{\partial r} .
$$

These quantities were computed using $v_{T} / w_{m} r_{m}=-f_{u w} / f_{w}^{\prime}$ and $D_{T} / w_{m} r_{m}=-f_{u b} / f_{b}^{\prime}$, where the similarity functions $f_{\chi}$ are the averages of those presented in Fig. 4 and the prime denotes differentiation with respect to $\eta$. The results are shown in Figs. 12(a) and 12(b) for the jet and plume, respectively. The radial distributions of $\nu_{T}$ and $D_{T}$ have a similar shape, with $D_{T}$ systematically higher than $v_{T}$ for both the jet and the plume. The values for $v_{T}$ and $D_{T}$ are slightly higher for the plume than for the jet. 

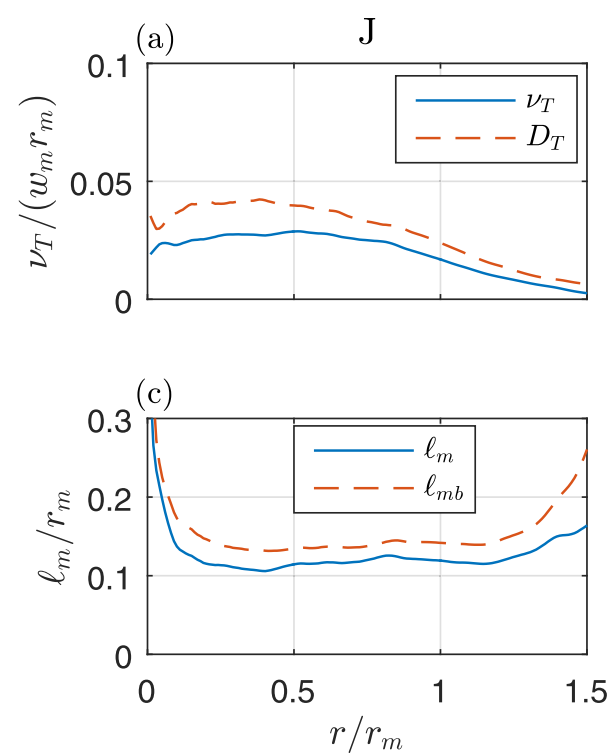

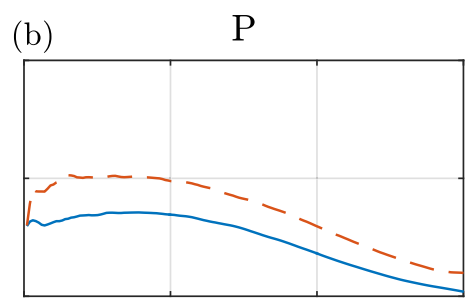

(d)

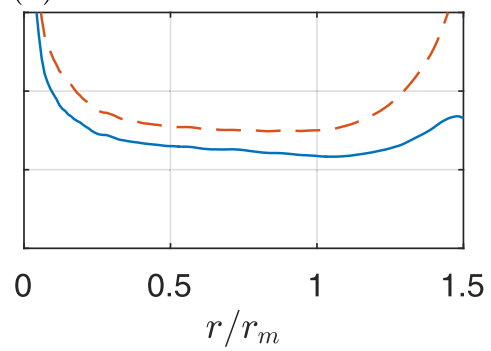

FIG. 12. Radial profiles of $v_{T}$ and $D_{T}$ for (a) the jet and (b) the pure plume. Radial mixing length radial profiles for (c) the jet and (d) the pure plume.

The profiles for $\nu_{T}$ and $D_{T}$ show substantial variations over the interval $0<r / r_{m}<1$. A Prandtl mixing length model [42] with mixing lengths for momentum and buoyancy of the form $\ell_{m}=a_{w} r_{m}$ and $\ell_{m b}=a_{b} r_{m}$, resulting in

$$
\frac{v_{T}}{w_{m} r_{m}}=a_{w}^{2}\left|f_{w}^{\prime}\right|, \quad \frac{D_{T}}{w_{m} r_{m}}=a_{b}^{2}\left|f_{w}^{\prime}\right|
$$

provides values of $\ell_{m} / r_{m} \equiv a_{w}$ and $\ell_{m b} / r_{m} \equiv a_{b}$ that are roughly constant in the core region [Figs. 12(c) and 12(d)]. Very close to the centerline, the mixing length becomes very large because $\left|f_{w}^{\prime}\right|$ and $\left|f_{b}^{\prime}\right|$ tend to zero. For $r / r_{m}>1$, the mixing length concept does not work well, which we attribute to intermittency effects associated with the plume edge. The typical values for $a_{w}$ and $a_{b}$ over the region $0.3<r / r_{m}<1.0$ are presented in Table I. Estimates of the mixing length show remarkable agreement with the experimental results recently presented by Ezzamel et al. [25], who estimated the Eulerian integral length scale of the two-point velocity statistics (their Fig. 15). In particular, note that the measurements revealed almost constant values of the Eulerian integral length in the core of the plume, for both jets and plumes.

The turbulent Prandtl number $\operatorname{Pr}_{T}$ is a quantity of great relevance because of its extensive use in turbulence modeling. By substituting (24) into (13), one obtains

$$
\operatorname{Pr}_{T}=\frac{v_{T}}{D_{T}}=\frac{f_{u w}}{f_{u b}} \frac{f_{b}^{\prime}}{f_{w}^{\prime}}
$$

Thus, $\operatorname{Pr}_{T}$ can be thought of as the product of two ratios: (i) the ratio of the radial turbulent fluxes $f_{u w} / f_{u b}$ and (ii) the ratio of gradients of the mean buoyancy and velocity $f_{b}^{\prime} / f_{w}^{\prime}$. The turbulent Prandtl number, plotted in Fig. 13, is almost constant over the entire cross section with values in the range 0.6-0.8. The average value $\left\langle\operatorname{Pr}_{T}\right\rangle$ over the interval $0.3<r / r_{m}<1.0$ is 0.72 for the jet simulation and 0.67 for the plume simulation (see also Table I). Thus, the estimates of $\left\langle\operatorname{Pr}_{T}\right\rangle$ are remarkably close, despite the effect of buoyancy on the plume's behavior. Shown in Fig. 13(b) is the ratio $f_{b}^{\prime} / f_{w}^{\prime}$. For the plume, the ratio is approximately unity, but for the jet it is significantly lower due to the fact that $\theta_{m}<1$ and thus $\varphi>1$. The ratio $f_{u w} / f_{u b}$, shown in Fig. 13(c), is approximately 


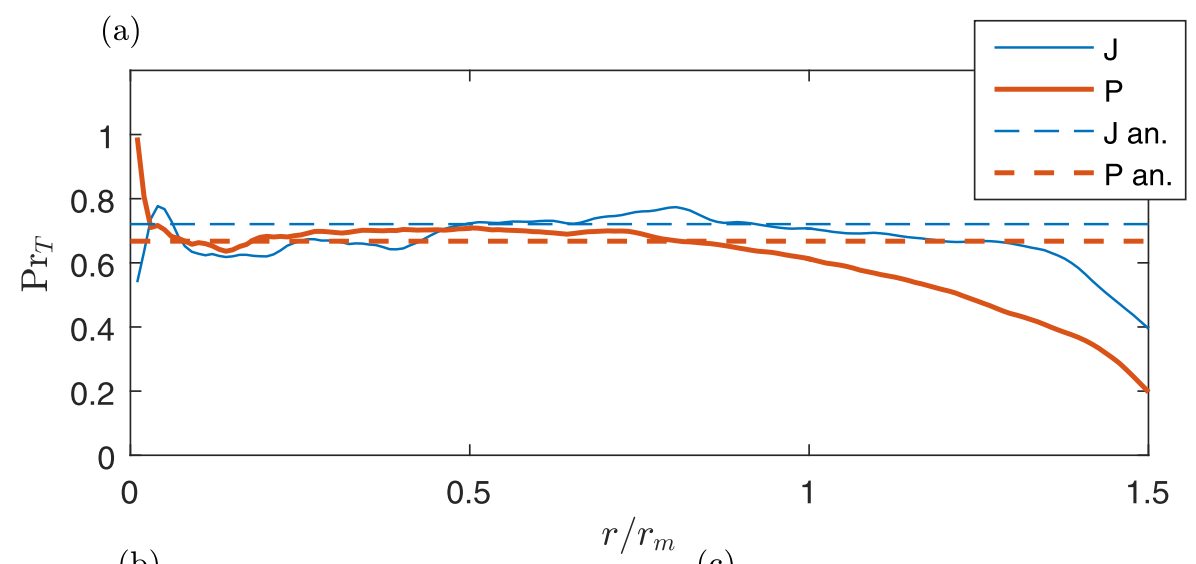

(b)

(c)
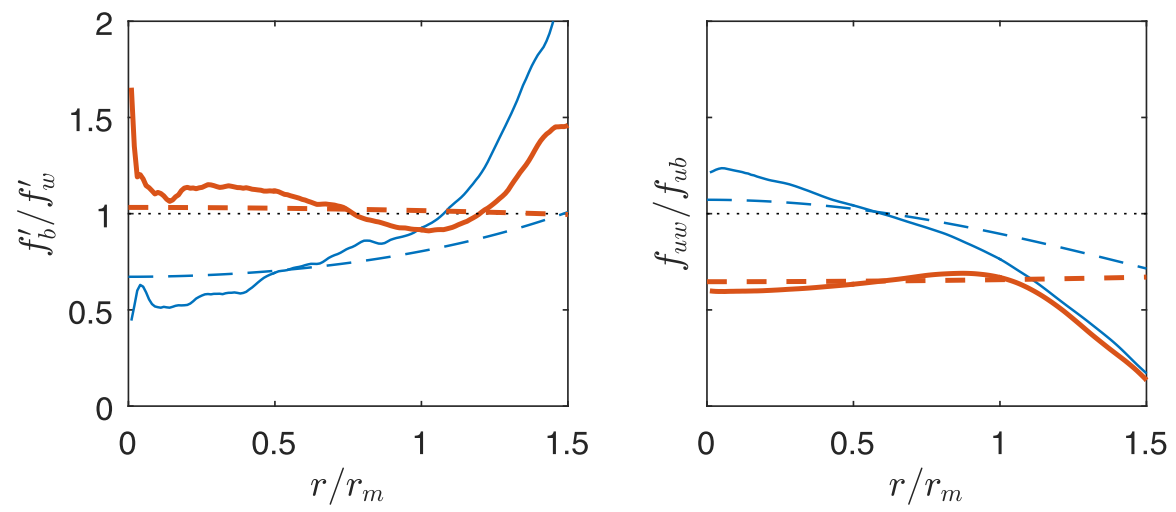

FIG. 13. Radial profiles of (a) the turbulent Prandtl number $\operatorname{Pr}_{T}$, (b) the ratio of the similarity functions $f_{b}^{\prime} / f_{w}^{\prime}$, and (c) $f_{u w} / f_{u b}$ (see the text). The solid lines show the DNS data and the dashed lines the analytical predictions (27).

constant for the plume with a value of about 0.6 . For the jet, $f_{u w} / f_{u b}$ decreases slowly with an average value of about 1 .

Thus, although $\operatorname{Pr}_{T}$ is very similar for plumes and jets, the reason is different: for jets it is caused primarily by $f_{b}^{\prime} / f_{w}^{\prime}$, which is associated with the ratio of widths $\varphi$, and for the plume primarily by the turbulent flux ratio $f_{u w} / f_{u b}$ (see also Fig. 4). This can be made explicit by evaluating the ratios [by substituting the Gaussian profiles for $f_{w}=\bar{w} / w_{m}$ and $f_{b}=\bar{b} / b_{m}$ (20)] into (24) and (25), resulting in

$$
\frac{f_{b}^{\prime}}{f_{w}^{\prime}}=\frac{1}{\varphi^{4}} \exp \left(-\frac{\varphi^{2}-1}{\varphi^{2}} \frac{r^{2}}{r_{m}^{2}}\right), \quad \frac{f_{u w}}{f_{u b}}=\varphi^{4} \frac{a_{w}^{2}}{a_{b}^{2}} \exp \left(\frac{\varphi^{2}-1}{\varphi^{2}} \frac{r^{2}}{r_{m}^{2}}\right)
$$

noting that $\left(\varphi^{2}-1\right) / \varphi^{2}=\left(2-2 \theta_{m}\right) /\left(2-\theta_{m}\right)$. The product of these two terms evaluates to $\left\langle\operatorname{Pr}_{T}\right\rangle=$ $a_{w}^{2} / a_{b}^{2}$, consistent with (25). Equation (27) shows that the amplitude of the ratio $f_{b}^{\prime} / f_{w}^{\prime}$ is solely determined by the value of $\varphi$. The amplitude of the ratio $f_{u w} / f_{u b}$ is determined both by $\varphi$ and the ratio of mixing lengths $a_{w} / a_{b}$. The theoretical predictions of (27), using parameter values for $a_{w}$ and $a_{b}$ from Table I and $\theta_{m}$ from Table III, are plotted in Fig. 13 with dashed lines. The results agree quite well in the interval $0<r / r_{m}<1$, in terms of both the amplitude and the trend. Near the plume edge, it is clear that the mixing lengths and Gaussian distributions do not describe the behavior. 
Previous authors [25] have suggested that a spatially averaged (over the radial plume section) turbulent Prandtl number $\left\langle\operatorname{Pr}_{T}\right\rangle$ can be inferred from the ratio of the plume radii $r_{m}$ and $r_{b}$, estimated through a Gaussian fit of the radial profiles of mean vertical velocity and buoyancy, respectively. For jets this approach is valid because, to leading order, the scalar field and the vertical velocity field essentially obey the same similarity equations, which state that radial mixing must balance the divergence in the vertical flux. As noted previously [43], the ratio of $r_{m}$ and $r_{b}$ can be obtained via the substitution of Gaussian profiles into the similarity equations. Evaluation of the resulting balance on the centerline of the flow allows one to relate $D_{T}$ to $r_{b}$ and $v_{T}$ to $r_{m}$. Equivalently, one can view the problem in a moving frame of reference, in which $z^{2} \propto t$, and apply the classical relation for diffusion, which predicts that $r_{b} \propto \sqrt{t D_{T}}$ and $r_{m} \propto \sqrt{t v_{T}}$. Both approaches result in $\left\langle\operatorname{Pr}_{T}\right\rangle=\varphi^{-2}$. For jets, we observe that $\varphi \approx 1.1$ and therefore would expect $\left\langle\operatorname{Pr}_{T}\right\rangle \approx 0.8$, which is reasonably consistent with Fig. 13(a). In the case of plumes, however, the analysis described above is not appropriate, unless one accounts for the additional term arising from buoyancy in the governing momentum equation. Indeed, our results indicate values of $\left\langle\operatorname{Pr}_{T}\right\rangle$ that are systematically lower than unity in plumes [see, e.g., Fig. 13(a)], in spite of the fact that $\varphi \approx 1$.

\section{CONCLUSION}

The dynamics and transport properties of a turbulent pure jet, a pure plume, and a forced plume were examined using high-fidelity direct numerical simulations. The motivation for this work, the numerical analog of the experimental study by Ezzamel et al. [25], was specifically to shed light on the physical processes linking turbulent transport and entrainment.

The detailed spatial resolution of the DNS allowed the effectiveness of turbulent transport to be quantified, e.g., via turbulent diffusion coefficients and the dilution of fluid in the plume or jet with the ambient. For the forced plume, within which the flow dynamically adjusts towards a pure-plume behavior asymptotically with height, of particular relevance was the vertical variation of the entrainment coefficient $\alpha$, numerous models having been proposed to capture this variation. Our results support the Priestley-Ball [3] entrainment model (12) and show that, beyond a nearsource region (specifically for $z / r_{0} \gtrsim 20$ ), the entrainment coefficient is a function only of the local Richardson number.

By decomposing $\alpha$ [see (22)] into contributions due to turbulence production, to buoyancy, and to shape effects, we show that the production of turbulence due to shear (as represented by the dimensionless quantity $\delta_{m}$ ) is practically identical for jets and for plumes, which is indeed the assumption underlying (12). Moreover, since the turbulent component of entrainment has been shown to be unaltered by buoyancy [26], this confirms that $\alpha$ is larger for plumes than for jets due to entrainment associated with mean flow processes.

The fact that the production of turbulence due to shear takes approximately the same value for jets and plumes suggests that their turbulence structure is quite similar, despite the absence of buoyancy in a jet. The second-order statistics $\overline{u^{\prime} u^{\prime}}, \overline{v^{\prime} v^{\prime}}$, and $\overline{w^{\prime} w^{\prime}}$ indeed suggest that the turbulence levels are very similar. The invariance of the turbulence anisotropy tensor confirms that turbulence in the core region of a jet or plume is practically indistinguishable. There is, however, evidence of clear distinctions between the structure of a jet and a plume. For example, while there is a transition from rodlike to disklike turbulence moving radially outward from the centerline, this transition occurs closer to the centerline in a plume; these distinctions are believed to be linked with vertical velocity gradients $\partial \bar{w} / \partial z$. Further differences between jets and plumes exist in the second-order scalar statistics, such as $\overline{w^{\prime} b^{\prime}}$ and $\overline{b^{\prime} b^{\prime}}$. Analysis of the budgets for these quantities would indicate how such differences can exist between flows whose dynamics are similar and would therefore make a valuable contribution to an overall understanding of turbulence in free-shear flows.

In agreement with existing measurements, the turbulent Prandtl number is found to be almost identical for jets and plumes, taking a value of $\left\langle\operatorname{Pr}_{T}\right\rangle=0.7$. However, by writing this quantity as the ratio of turbulent fluxes and radial gradients of mean quantities, it becomes evident that for jets, the 
value of $\left\langle\operatorname{Pr}_{T}\right\rangle$ can be attributed to differences in the ratio of velocity to buoyancy profile widths $\varphi$, whereas for plumes, the value of $\left\langle\operatorname{Pr}_{T}\right\rangle$ is associated with the ratio of the turbulent radial transport of buoyancy and streamwise momentum.

The DNS data do not support the notion of similarity drift and we conjecture that the observed variations in profile widths between experiments are possibly a result of confinement or other deviations from ideal boundary conditions.

\section{ACKNOWLEDGMENTS}

We acknowledge the UK Turbulence Consortium (Grant No. EP/L000261/1) and an Engineering and Physical Sciences Research Council (EPSRC) ARCHER Leadership Grant for providing the computational resources required to carry out the computations. In addition, J.C. gratefully acknowledges funding from the EPSRC Doctoral Prize under Grant No. EP/M507878/1. P.S. gratefully acknowledges the support of Cambridge University's Engineering Department and Peterhouse College. Supporting data for this article are available on request.

\section{APPENDIX: AMBIENT-FLOW CORRECTION OF THE ESH DATA}

The purpose of this Appendix is twofold: (i) to correct the ESH data [25] for vertical variation in the ambient flow and (ii) to present the experimental data in terms of the notation used in this paper. A significant part of the ESH work was associated with the analysis of $\alpha(z)$. The $z$ dependence of $\alpha$ was determined in two ways: (i) via volume conservation (6) and (ii) via the entrainment relation (22) considering mean contributions and self-similarity only, assuming Gaussian profiles $\left(\gamma_{m}=4 / 3\right)$ :

$$
\alpha=-\frac{3}{8} \delta_{m}+\left(1-\frac{3}{4} \theta_{m}\right) \mathrm{Ri}
$$

In Ref. [25] this relation was presented in terms of the relative plume width $\varphi=\sqrt{2 / \theta_{m}-1}$, the effective eddy viscosity $\left\langle\hat{v}_{T}\right\rangle=-\delta_{m} / 8 \sqrt{2}$, and the flux balance parameter $\Gamma=5 \mathrm{Ri} / 8 \alpha_{p}$ (note that $\beta_{g}=1$ as only means are considered), i.e., as

$$
\alpha_{G}=3\left\langle\hat{v}_{T}\right\rangle+\left(2 \varphi^{2}-1\right) \frac{2 \alpha_{p G} \theta_{m}}{5} \Gamma
$$

Here $\alpha_{G}=\alpha / \sqrt{2}$ is the Gaussian entrainment coefficient and $\alpha_{p G}=\alpha_{p} / \sqrt{2}$ the Gaussian entrainment coefficient for a pure plume. The prefactor for $\left\langle\hat{v}_{T}\right\rangle$ is a factor 2 larger than reported in Ref. [25]. Furthermore, the factor $\theta_{m}$ in the buoyancy contribution was not present in Ref. [25]; this is caused by the inclusion of $\beta_{g}$ and $\theta_{m}$ in the flux balance parameter $\Gamma$ (15). Indeed, denoting the classical flux balance parameter [5] by $\Gamma^{*}=5 F Q^{2} / 8 \alpha_{p} M^{5 / 2}$, we have $\Gamma^{*}=\beta_{g} \theta_{m} \Gamma$.

As discussed in Ref. [25], the measurements revealed a small but significant flow in the ambient, caused by (i) the diffusion of heat from the warm-air plume source along the horizontal rigid wooden base plate within which the plume nozzle was mounted, giving rise to vertical convective motion, and (ii) the seeding of the ambient with a stage smoke generator. Indeed, the background mean motion, whose vertical velocity we denote by $\Delta w$, was clearly captured by particle image velocimetry (PIV) fields when measuring velocities away from the plume perimeter in the lower regions of the domain, a region where the plume width was significantly smaller than the lateral extent of the PIV field. Measurements indicated $\Delta w \approx 0.15 \mathrm{~ms}^{-1}$ close to the source (whose radius is denoted $r_{0}$ ) for the jetlike, the forced, and the pure-plume experiments, referred to as J, F, and P, respectively. However, at larger vertical distances above the source, the size of the PIV field did not permit measurement of the (now significantly wider) plume or the ambient far beyond the plume perimeter. In Ref. [25] it was therefore assumed that the background motion was uniform throughout the domain; hence $\Delta w=0.15 \mathrm{~ms}^{-1}$ was subtracted from the mean vertical velocities before fitting the radial profiles 
(a)

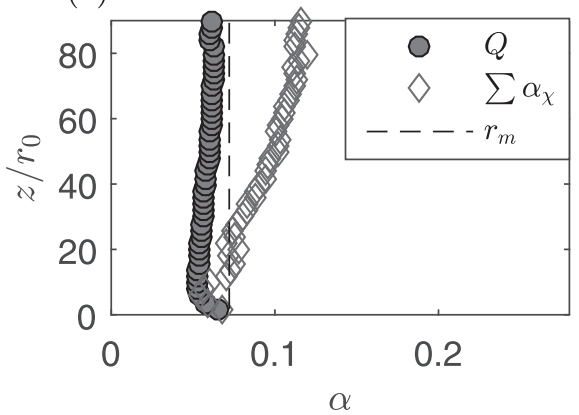

(c)

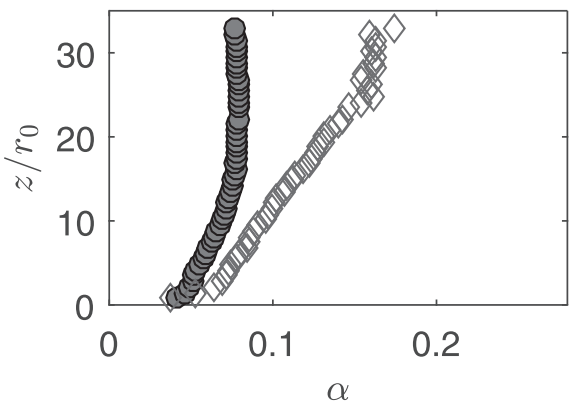

(e)

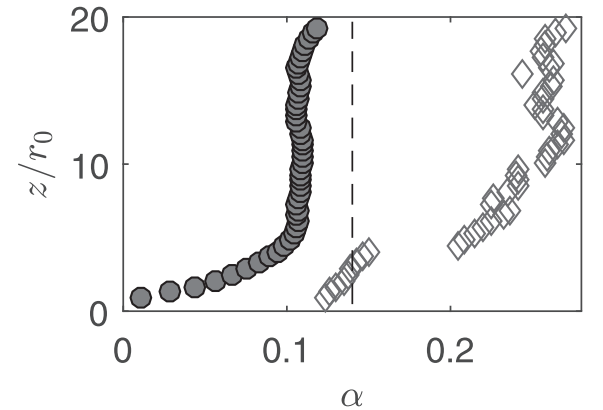

(b)

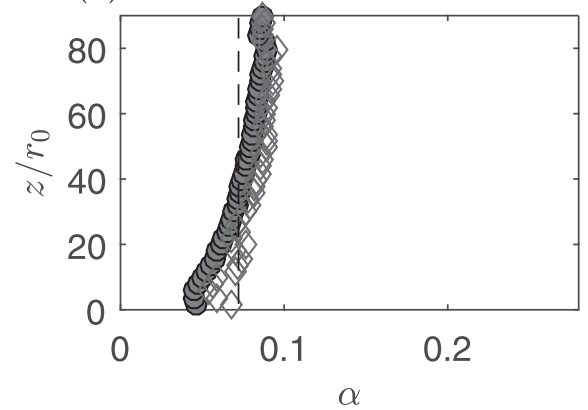

(d)

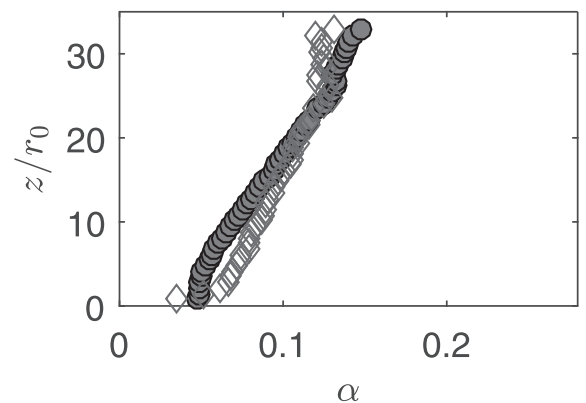

(f)

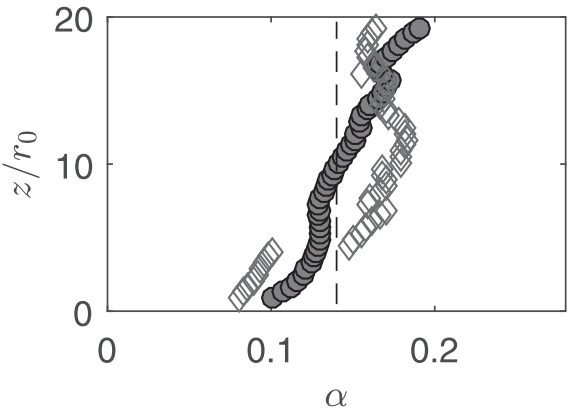

FIG. 14. Variation of entrainment coefficient as a function of distance above the source. Comparison of entrainment coefficient estimated from $r_{m}$, the volume flux balance (6), and from the entrainment relation (A2) for (a) and (b) J, (c) and (d) F, and (e) and (f) P with (a), (c), and (e) $\Delta w=0.15 \mathrm{~ms}^{-1}$ and (b), (d), and (f) $\Delta w=0.15\left(z / z_{0}\right)^{-1 / 3} \mathrm{~ms}^{-1}$.

with a Gaussian curve of the form

$$
\frac{\bar{w}(r, z)}{w_{g}(z)}=\exp \left(\frac{-r^{2}}{r_{g}^{2}(z)}\right)
$$

where $w_{g}=2 w_{m}$ denotes the plume centerline velocity and $r_{g}=r_{m} / \sqrt{2}$ the Gaussian plume radius.

Figures 14(a), 14(c), and 14(e) show the J, F, and P estimates for $\alpha$ from [25] in the current notation. Indicated with the dashed line in Figs. 14(a) and 14(c) is an estimate for $\alpha$ inferred from $r_{m}(z)$ (using the relations for $r_{m}$ in Table II). All three estimates of $\alpha$ should formally provide the same value for $\alpha$. For the DNS data, this is clearly the case (Fig. 10), but experiments are much more difficult to control, particularly the boundary conditions. The measurement data show a 

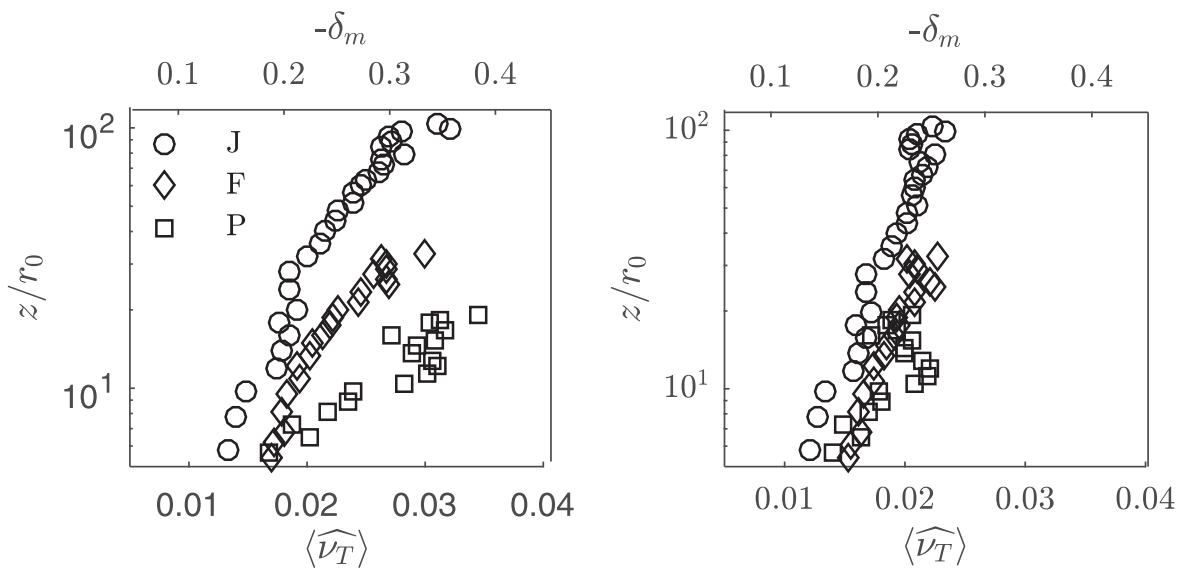

FIG. 15. Vertical evolution of $\delta_{m}$ for $\mathrm{J}, \mathrm{F}$, and $\mathrm{P}$ with (a) $\Delta w=0.15 \mathrm{~ms}^{-1}$ and (b) $\Delta w=$ $0.15\left(z / z_{0}\right)^{-1 / 3} \mathrm{~ms}^{-1}$.

large discrepancy between the $Q$-based estimate for $\alpha$ and the one obtained from the entrainment relation (A2). This difference points to a mismatch in either the momentum balance or the mean kinetic energy balance, which can be traced back to the background flow in the ambient.

In what follows we show that the differences between our estimates for $\alpha$ can be significantly reduced by using a background motion whose magnitude is progressively reduced with distance from the source. As a consequence of the convection above the base plate, the plumes studied developed in a weak background velocity field that we would expect to scale as $\Delta w \sim z^{-1 / 3}$, i.e., the plume effectively developed within a weaker plume rising from the base plate. By applying a background correction of the form $\Delta w=0.15\left(z / z_{0}\right)^{-1 / 3}$, where $z_{0}$ is the distance from the plate where the ambient vertical velocity was $0.15 \mathrm{~m} / \mathrm{s}$, the three estimates of $\alpha$ exhibit an improved agreement, as shown in Figs. 14(b), 14(d), 14(f); all estimates are in reasonably good agreement with each other.

The method by which $\delta_{m}$ has been calculated for the entrainment relation data (A2) is performed differently than in Ref. [25]. Indeed, upon close inspection of the experimental radial profiles of the Reynolds stress $\overline{u^{\prime} w^{\prime}}$, in Ref. [25] the gradient diffusion hypothesis led to a systematic overestimation of $\delta_{m}$. As in Ref. [25], the $\overline{u^{\prime} w^{\prime}}$ profile is fitted to a function of the form

$$
\frac{\overline{u^{\prime} w^{\prime}}}{w_{g}^{2}}=2\left\langle\hat{v}_{T}\right\rangle \frac{r}{r_{g}} \exp \left(-\frac{r^{2}}{r_{g}^{2}}\right),
$$

which follows from the substituting the Gaussian velocity profile (A3) into the gradient-diffusion hypothesis (24) using a constant (in $r$ ) eddy viscosity $\left\langle v_{T}\right\rangle=w_{g} r_{g}\left\langle\hat{v}_{T}\right\rangle$. However, we now consider $r_{g}$ as a free parameter [not necessarily fixed by the value provided by the fit of (A3)] and calculate $\left\langle\hat{v}_{T}\right\rangle$ based on the value of $r_{g}$ for which the least-squares error between the measurements and (A4) is minimized.

By substituting (A3) and (A4) into the definition for $\delta_{m}$, it immediately follows that $\delta_{m}=$ $-8 \sqrt{2}\left\langle\hat{v}_{T}\right\rangle$; the corrected values for both $\left\langle\hat{v}_{T}\right\rangle$ and $\delta_{m}$ are shown in Fig. 15(b). For all three releases, the values for $\delta_{m}$ are now reasonably consistent, although there is a clear increasing trend with $z$ that is not consistent with fully self-similar behavior (in which case $\delta_{m}$ is expected to be constant). Nevertheless, the data are much more consistent than the original ambient-flow correction estimate shown in Fig. 15(a). The data shown in Figs. 14(b), 14(d), 14(f), and 15(b) were used to provide the input to Table 3 in Ref. [26]. 
[1] J. C. R. Hunt, Industrial and environmental fluid mechanics, Annu. Rev. Fluid Mech. 23, 1 (1991).

[2] Y. Zel'dovich, The asymptotic laws of freely-ascending convective flows, Zh. Eksp. Teor. Fiz. 7, 1463 (1937).

[3] C. Priestley and F. K. Ball, Continuous convection from an isolated source of heat, Q. J. R. Meteor. Soc. 81, 144 (1955).

[4] B. R. Morton, G. I. Taylor, and J. S. Turner, Turbulent gravitational convection from maintained and instantaneous sources, Proc. R. Soc. London Ser. A 234, 1 (1956).

[5] B. R. Morton, Forced plumes, J. Fluid Mech. 5, 151 (1959).

[6] P. N. Papanicolaou and E. J. List, Investigations of round vertical turbulent buoyant jets, J. Fluid Mech. 195, 341 (1988).

[7] N. R. Panchapakesan and J. L. Lumley, Turbulence measurements in axisymmetric jets of air and helium. Part 1. Air jet, J. Fluid Mech. 246, 197 (1993).

[8] A. Shabbir and W. K. George, Experiments in a round turbulent buoyant plume, J. Fluid Mech. 275, 1 (1994).

[9] H. Wang and A. W.-K. Law, Second-order integral model for a round turbulent buoyant jet, J. Fluid Mech. 459, 397 (2002).

[10] M. V. Pham, F. Plourde, and K. S. Doan, Direct and large-eddy simulations of a pure thermal plume, Phys. Fluids 19, 125103 (2007).

[11] F. Plourde, M. V. Pham, S. D. Kim, and S. Balachandar, Direct numerical simulations of a rapidly expanding thermal plume: Structure and entrainment interaction, J. Fluid Mech. 604, 99 (2008).

[12] E. List, Turbulent jets and plumes, Annu. Rev. Fluid Mech. 14, 189 (1982).

[13] A. W. Woods, Turbulent plumes in nature, Annu. Rev. Fluid Mech. 42, 391 (2010).

[14] G. R. Hunt and T. S. van den Bremer, Classical plume theory: 1937-2010 and beyond, IMA J. Appl. Math. 76, 424 (2011).

[15] G. R. Hunt and N. B. Kaye, Lazy plumes, J. Fluid Mech. 533, 329 (2005).

[16] J. Craske, A. Debugne, and M. van Reeuwijk, Shear-flow dispersion in turbulent jets, J. Fluid Mech. 781, 28 (2015).

[17] E. Kaminski, S. Tait, and G. Carazzo, Turbulent entrainment in jets with arbitrary buoyancy, J. Fluid Mech. 526, 361 (2005).

[18] G. I. Taylor, Dynamics of a mass of hot gas rising in air, US Atomic Energy Commission, Los Alamos National Laboratory Report No. 236, 1945 (unpublished).

[19] G. K. Batchelor, Heat convection and buoyancy effects in fluids, Q. J. R. Meteor. Soc. 80, 339 (1954).

[20] J. Turner, Turbulent entrainment: the development of the entrainment assumption, and its application to geophysical flows, J. Fluid Mech. 173, 431 (1986).

[21] G. Carazzo, E. Kaminski, and S. Tait, The route to self-similarity in turbulent jets and plumes, J. Fluid Mech. 547, 137 (2006).

[22] E. J. List and J. Imberger, Turbulent entrainment in buoyant jets and plumes, J. Hydraul. Div. ASCE 99, 1461 (1973).

[23] H. B. Fischer, E. J. List, R. C. Koh, J. Imberger, and N. H. Brooks, Mixing in Inland and Coastal Waters (Academic Press, New York, 1979).

[24] D. G. Fox, Forced plume in a stratified fluid, J. Geophys. Res. 75, 6818 (1970).

[25] A. Ezzamel, P. Salizzoni, and G. R. Hunt, Dynamical variability of axisymmetric buoyant plumes, J. Fluid Mech. 765, 576 (2015).

[26] M. van Reeuwijk and J. Craske, Energy-consistent entrainment relations for jets and plumes, J. Fluid Mech. 782, 333 (2015).

[27] C. Chen and W. Rodi, Vertical Turbulent Buoyant Jets-A Review of Experimental Data (Pergamon, Oxford, 1980).

[28] L. P. Chua and R. A. Antonia, Turbulent Prandtl number in a circular jet, Int. J. Heat Mass Transf. 33, 331 (1990).

[29] G. R. Hunt and N. B. Kaye, Virtual origin correction for lazy turbulent plumes, J. Fluid Mech. 435, 377 (2001). 
[30] W. K. George, R. Alpert, and F. Tamanini, Turbulence measurements in an axisymmetric buoyant plume, Int. J. Heat Mass Transf. 20, 1145 (1977).

[31] H. Rouse, C. S. Yih, and H. W. Humphreys, Gravitational convection from a boundary source, Tellus 4, 201 (1952).

[32] B. J. Devenish, G. G. Rooney, and D. J. Thomson, Large-eddy simulation of a buoyant plume in uniform and stably stratified environments, J. Fluid Mech. 652, 75 (2010).

[33] J. Craske and M. van Reeuwijk, Energy dispersion in turbulent jets. Part 1. Direct simulation of steady and unsteady jets, J. Fluid Mech. 763, 500 (2015).

[34] J. Craske and M. van Reeuwijk, Robust and accurate open boundary conditions for incompressible turbulent jets and plumes, Comput. Fluids 86, 284 (2013).

[35] J. Craske and M. van Reeuwijk, Energy dispersion in turbulent jets. Part 2. A robust model for unsteady jets, J. Fluid Mech. 763, 538 (2015).

[36] J. Craske and M. van Reeuwijk, Generalised unsteady plume theory, J. Fluid Mech. 792, 1013 (2016).

[37] J. H. Hussein, S. P. Capp, and W. K. George, Velocity measurements in a high-Reynolds-number, momentum conserving, axisymetric jet, J. Fluid Mech. 258, 31 (1994).

[38] S. B. Pope, Turbulent Flows (Cambridge University Press, Cambridge, 2000).

[39] F. Kuznik, G. Rusaouen, and J. Brau, Experimental study of turbulent structures in a non isothermal horizontal jet issuing from a round nozzle distanced from a wall, Int. J. Ventil. 10, 277 (2011).

[40] M. Woodhouse, J. Phillips, and A. Hogg, Unsteady turbulent buoyant plumes, J. Fluid Mech. 794, 595 (2016).

[41] P. F. Linden, in Perspectives in Fluid Dynamics: A Collective Introduction to Current Research, edited by G. K. Batchelor, H. K. Moffatt, and M. G. Worster (Cambridge University Press, Cambridge, 2003), Chap. 8, p. 289.

[42] H. Schlichting, Boundary Layer Theory (McGraw-Hill, New York, 1960).

[43] J. Chen and E. J. List, Proceedings of the 1976 ICHMT Seminar on Turbulent Buoyant Convection (unpublished), p. 171. 